\title{
MULTISCALE REVERSE-TIME-MIGRATION-TYPE IMAGING USING THE DYADIC PARABOLIC DECOMPOSITION OF PHASE SPACE
}

\author{
FREDRIK ANDERSSON*, MAARTEN V. DE HOOP ${ }^{\dagger}$, AND HERWIG WENDT ${ }^{\ddagger}$
}

\begin{abstract}
We develop a representation of reverse-time migration in terms of Fourier integral operators the canonical relations of which are graphs. Through the dyadic parabolic decomposition of phase space, we obtain the solution of the wave equation with a boundary source and homogeneous initial conditions using wave packets. On this basis, we develop a numerical procedure for the reversetime continuation from the boundary of scattering data and for RTM migration. The algorithms are derived from those we recently developed for the discrete approximate evaluation of the action of Fourier integral operators and inherit from their conceptual and numerical properties.
\end{abstract}

1. Introduction. Reflection seismology is a commonly used method to study the properties of Earth's subsurface in geophysical exploration. Point sources are placed on Earth's surface which generate acoustic waves in the subsurface that are reflected where the medium properties vary discontinuously. These reflections are recorded at Earth's surface by (arrays of) point receivers. The goal of seismic imaging is to reconstruct the singular variations in medium properties from the reflected waves recorded at the surface [10, 4, 29. The most common formulation for seismic inverse scattering takes the form of a linearized inverse problem for the medium coefficient in the acoustic wave equation, where the linearization is performed about a smoothly varying background. Here, the background model is assumed to be known. However, via a formulation as a separable inverse problem one can also proceed with determining this background model. The linearization defines a single scattering operator that maps the coefficient contrast to the data, i.e., the restriction of the scattered wave field to the acquisition set. The adjoint defines the process of imaging.

We consider reverse-time migration (RTM) 23, 32, 19, 3, 28, and the RTMbased inverse scattering transform developed and analyzed by Op't Root et al. 20. Through an appropriate formulation based on wave field continuation [16, we arrive at a representation of RTM in terms of a Fourier Integral Operator (FIO) associated with a canonical graph. Indeed, we use such a representation. The key contribution of this paper lies in the development of an algorithm for the solution of the wave equation with a boundary source and homogeneous initial conditions using the dyadic parabolic decomposition of phase space in the limit of fine scales. This algorithm is then composed with an imaging condition to yield the RTM-type imaging or inverse scattering. We explicitly admit the formation of caustics.

Viewing wave packets as localized plane waves, our approach has connections to methods in which one designs sources that favor (directional) illumination of particular subdomains of the subsurface. We mention plane-wave migration and beam-wave migration. In plane-wave migration one synthesizes plane-wave source experiments 33. Given a plane-wave source one can then introduce tilted coordinates to carry out the wave field extrapolation with a limited accuracy propagator 24]. In beamwave migration, Brandsberg-Dahl and Etgen [5] use a rotating coordinate system and

\footnotetext{
* Mathematics LTH, Centre for Mathematical Sciences, Lund Institute of Technology, Lund University, Sweden (fa@maths.lth.se)

${ }^{\dagger}$ Department of Mathematics, Purdue University, West Lafayette, IN 47907, USA (mdehoop@purdue.edu).

${ }^{\ddagger}$ CNRS, Institut de Recherche en Informatique de Toulouse (IRIT), Toulouse, France (herwig.wendt@irit.fr)
} 
essentially couple wave field methods with band-limited properties to ray-geometric methods. Furthermore, we mention the use of coherent states in this context by Albertin et al. [1. Instead of tilted coordinates, one can use curvilinear coordinates in combination with a paraxial propagator [22]; the curvilinear coordinates may be generated as geodesics initiated from a point source or a plane wave. We note, however, that these methods are downward-continuation based whereas our approach is based on reverse-time continuation; also, we decompose the data although we could incorporate a synthesis of wave packet sources as well.

Our numerical solution is derived from the algorithm that we developed for FIOs 2]. Computational efficiency arises from organizing the decomposition and propagation by directions associated with frequency boxes instead of individual wave packets. The superposition of wave packets is complete and their propagation, as well as the corresponding imaging, converges in the limit of fine scales in smooth velocity models. Our formulation is insensitive to specific choices of (absorbing) boundary conditions, which is in contrast to PDE based solutions, including beam-wave migration. Moreover, it naturally conveys angular information which can be used in the imaging process, for instance, for computing restricted angle transforms. Candès, Demanet \& Ying developed a fast butterfly algorithm for the application of Fourier integral operators associated with canonical graphs [9], which presents an interesting alternative to the propagation component of our algorithm.

Our algorithm is particularly well suited for application to (limited aperture) seismic array data providing a way of partial (in phase space) imaging possibly with a small set of sources. Moreover, if one need not generate a 'global' image, we do not need to evaluate the relevant wave field solutions at 'all' times, unlike algorithms based on numerically solving the wave equation, which enables computationally efficient target-oriented imaging. Target-oriented imaging can be used effectively, for example, with available arrays and earthquakes in studying heterogeneities and discontinuities in Earth's mantle [18, 31]. A key element of our algorithm is finding a low-rank separated representation of the amplitude of the relevant FIO, which we do using Prolate Spheroidal Wave Functions (PSWFs) 2]. Demanet \& Ying 12 proposed a method of finding such a representation based on the randomized sampling algorithm for constructing factorizations for low-rank matrices.

Our algorithm involves the propagation of high-frequency waves. To compare its complexity with the computational complexities of RTM algorithms based on numerically solving the wave equation, one can essentially compare the complexities of the backpropagation of a boundary source (from single-source data). Considering (backward) solving the wave equation in dimension $n$ on a grid of side length $N$ and a propagation time of order $\mathcal{O}(1)$, the CFL condition implies that the time step is of order $\mathcal{O}(1 / N)$. The $\mathcal{O}(N)$ applications of a short-time propagator, with a presumed complexity $\mathcal{O}\left(N^{n}\right)$, then yields a complexity $\mathcal{O}\left(N^{n+1}\right)$. The time step in our algorithm is $\mathcal{O}(1)$ in principle, while the application of the Fourier integral operator representing the propagator is $\mathcal{O}\left(N^{n} \log N\right)$ per frequency box (see [2]); the number of time steps to be computed is affected by the size of the target. Demanet \& Ying [12] already pointed out the time upscaling of an approach to wave propagation using a FIO.

The outline of the paper is as follows. In Section 2, we summarize the parametrix construction of the wave equation, and introduce the relevant Hamilton system and linearized Hamilton-Jacobi equations describing the geometry of the imaging process. In Section 3 we formulate reverse-time continuation from the boundary and obtain a 
particular oscillatory integral representation for the kernel of this process, to which the algorithm for FIOs that we developed in an earlier paper applies. Subsection 3.3 contains this key new result, and Subsection 3.4 its computational counterpart. These are also the main components of the asymptotic form of the RTM-based inverse scattering transform and imaging algorithm, which we develop in Section 4 In Section 5 . we give numerical examples of reverse-time continuation and inverse scattering also in the presence of caustics. We end with a discussion in Section 6 .

Dyadic parabolic decomposition of phase space. We briefly discuss the (co)frame of curvelets and wave packets [8, 14, 25. We will implicitly suppose that the data are decomposed into wave packets below, and we will develop wave packet based algorithms with accuracy $\mathcal{O}\left(2^{-k / 2}\right)[2$.

Let $u \in L^{2}\left(\mathbb{R}^{n}\right)$ represent a (seismic) velocity field, and let $\hat{u}(\xi)=\int u(x) \exp [-\mathrm{i}\langle x, \xi\rangle] \mathrm{d} x$ be the Fourier transform. One begins with covering the positive $\xi_{1}$-axis $\left(\xi^{\prime}=\xi_{1}\right)$ by overlapping boxes of the form

$$
B_{k}=\left[\xi_{k}^{\prime}-\frac{L_{k}^{\prime}}{2}, \xi_{k}^{\prime}+\frac{L_{k}^{\prime}}{2}\right] \times\left[-\frac{L_{k}^{\prime \prime}}{2}, \frac{L_{k}^{\prime \prime}}{2}\right]^{n-1} .
$$

Here, both the centers $\xi_{k}^{\prime}$ and the side lengths $L_{k}^{\prime}, L_{k}^{\prime \prime}$ follow parabolic scaling

$$
\xi_{k}^{\prime} \sim 2^{k}, \quad L_{k}^{\prime} \sim 2^{k}, \quad L_{k}^{\prime \prime} \sim 2^{k / 2}, \quad \text { as } k \rightarrow \infty .
$$

Next, for each $k \geq 1$, let $\nu$ vary over a set of approximately $2^{k(n-1) / 2}$ uniformly distributed unit vector: ${ }^{1}$. Let $\Theta_{\nu, k}$ denote a choice of rotation matrix which maps $\nu$ to $e_{1}$, and

$$
B_{\nu, k}=\Theta_{\nu, k}^{-1} B_{k} .
$$

In the (co)frame construction, one encounters two sequences of smooth functions, $\hat{\chi}_{\nu, k}$ and $\hat{\beta}_{\nu, k}$, on $\mathbb{R}^{n}$, each supported in $B_{\nu, k}$, that form a copartition of unity

$$
\hat{\chi}_{0}(\xi) \hat{\beta}_{0}(\xi)+\sum_{k \geq 1} \sum_{\nu} \hat{\chi}_{\nu, k}(\xi) \hat{\beta}_{\nu, k}(\xi)=1,
$$

and satisfy the estimates

$$
\left|\left\langle\nu, \partial_{\xi}\right\rangle^{j} \partial_{\xi}^{\alpha} \hat{\chi}_{\nu, k}(\xi)\right|+\left|\left\langle\nu, \partial_{\xi}\right\rangle^{j} \partial_{\xi}^{\alpha} \hat{\beta}_{\nu, k}(\xi)\right| \leq C_{j, \alpha} 2^{-k(j+|\alpha| / 2)} .
$$

One now forms

$$
\hat{\psi}_{\nu, k}(\xi)=\rho_{k}^{-1 / 2} \hat{\beta}_{\nu, k}(\xi), \quad \hat{\varphi}_{\nu, k}(\xi)=\rho_{k}^{-1 / 2} \hat{\chi}_{\nu, k}(\xi),
$$

where $\rho_{k}$ is the volume of $B_{k}$. These functions satisfy the estimates

$$
\left.\forall N: \quad \begin{array}{l}
\left|\varphi_{\nu, k}(x)\right| \\
\left|\psi_{\nu, k}(x)\right|
\end{array}\right\} \leq C_{N} 2^{k(n+1) / 4}\left(2^{k}|\langle\nu, x\rangle|+2^{k / 2}\|x\|\right)^{-N} .
$$

To obtain a (co)frame, one introduces the integer lattice: $X_{j}:=\left(j_{1}, \ldots, j_{n}\right) \in \mathbb{Z}^{n}$, the dilation matrix

$$
D_{k}=\frac{1}{2 \pi}\left(\begin{array}{ll}
L_{k}^{\prime} & 0_{1 \times n-1} \\
0_{n-1 \times 1} & L_{k}^{\prime \prime} I_{n-1}
\end{array}\right), \quad \operatorname{det} D_{k}=(2 \pi)^{-n} \rho_{k},
$$

\footnotetext{
${ }^{1}$ By convention, we let $\nu(0)=e_{1}$ be aligned with the $\xi_{1}$-axis.
} 
and the points $x_{j}^{\nu, k}=\Theta_{\nu, k}^{-1} D_{k}^{-1} X_{j}$. The frame elements are now defined in the Fourier domain as

$$
\hat{\varphi}_{\gamma}(\xi)=\hat{\varphi}_{\nu, k}(\xi) \exp \left[-\mathrm{i}\left\langle x_{j}^{\nu, k}, \xi\right\rangle\right], \quad \gamma=(j, \nu, k), \quad k \geq 1,
$$

and similarly for $\hat{\psi}_{\gamma}(\xi)$. Thus, one obtains the transform pair

$$
u_{\gamma}=\int u(x) \overline{\psi_{\gamma}(x)} \mathrm{d} x, \quad u(x)=\sum_{\gamma} u_{\gamma} \varphi_{\gamma}(x)
$$

with the property that $\sum_{\gamma^{\prime}: k^{\prime}=k, \nu^{\prime}=\nu} u_{\gamma^{\prime}} \hat{\varphi}_{\gamma^{\prime}}(\xi)=\hat{u}(\xi) \hat{\beta}_{\nu, k}(\xi) \hat{\chi}_{\nu, k}(\xi)$ for each $\nu, k$.

2. Parametrix. Here, we summarize the parametrix construction for the wave equation. We consider the Cauchy initial value problem

$$
\begin{aligned}
{\left[\frac{\partial^{2}}{\partial t^{2}}+A\left(x, D_{x}\right)\right] u } & =0, & A\left(x, D_{x}\right) & =c(x) D_{x}^{2} c(x), \\
u(x, 0) & =0, & \frac{\partial u}{\partial t}(x, 0) & =h(x) ;
\end{aligned}
$$

we have normalized the pressure: $u=c^{-1} p$.

To evaluate the parametrix, we use the first-order system for $u$ that is equivalent to this wave equation,

$$
\frac{\partial}{\partial t}\left(\begin{array}{c}
u \\
\frac{\partial u}{\partial t}
\end{array}\right)=\left(\begin{array}{cc}
0 & 1 \\
-A\left(x, D_{x}\right) & 0
\end{array}\right)\left(\begin{array}{c}
u \\
\frac{\partial u}{\partial t}
\end{array}\right)
$$

This system can be decoupled, namely, by the matrix-valued pseudodifferential operators

$$
V\left(x, D_{x}\right)=\left(\begin{array}{cc}
1 & 1 \\
-\mathrm{i} B\left(x, D_{x}\right) & \mathrm{i} B\left(x, D_{x}\right)
\end{array}\right), \quad \Lambda\left(x, D_{x}\right)=\frac{1}{2}\left(\begin{array}{cc}
1 & \mathrm{i} B\left(x, D_{x}\right)^{-1} \\
1 & -\mathrm{i} B\left(x, D_{x}\right)^{-1}
\end{array}\right),
$$

where $B\left(x, D_{x}\right)=\sqrt{A\left(x, D_{x}\right)}$ is a pseudodifferential operator of order 1 .

The use of a general symbol $B$ in our presentation facilitates the extension of our algorithm to the imaging with elastic waves [6].

The principal symbol of $B\left(x, D_{x}\right)$ is given by $B^{\text {prin }}(x, \xi)=\sqrt{A^{\text {prin }}(x, \xi)}$. Then

$$
u_{ \pm}=\frac{1}{2} u \pm \frac{1}{2} \mathrm{i} B\left(x, D_{x}\right)^{-1} \frac{\partial u}{\partial t},
$$

satisfy the two first-order ("half wave") equations

$$
P_{ \pm}\left(x, D_{x}, D_{t}\right) u_{ \pm}=0
$$

where

$$
P_{ \pm}\left(x, D_{x}, D_{t}\right)=\frac{\partial}{\partial t} \pm \mathrm{i} B\left(x, D_{x}\right),
$$

supplemented with the initial conditions

$$
\left.u_{ \pm}\right|_{t=0}=h_{ \pm}, \quad h_{ \pm}= \pm \frac{1}{2} \mathrm{i} B\left(x, D_{x}\right)^{-1} h .
$$


We construct operators $S_{ \pm}(t)$ that solve the initial value problem 2.5), 2.7): $u_{ \pm}(y, t)=\left(S_{ \pm}(t) h_{ \pm}\right)(y)$. Then $u(y, t)=\left(\left[S_{+}(t)-S_{-}(t)\right] \frac{1}{2} \mathrm{i} B^{-1} h\right)(y)$. The operators $S_{ \pm}(t)$ are Fourier integral operators. Their construction is well known, see for example Duistermaat [17, Chapter 5]. Microlocally, the solution operator associated with 2.3. can be written in the matrix form

$$
S(t)=V\left(\begin{array}{cc}
S_{+}(t) & 0 \\
0 & S_{-}(t)
\end{array}\right) \Lambda
$$

in this notation, $S_{12}(t)=\left[S_{+}(t)-S_{-}(t)\right] \frac{1}{2} \mathrm{i} B^{-1}$.

For the later analysis, we introduce the operators $S(t, s)$ and $S_{ \pm}(t, s): S(t, s)$ solves the problem

$$
\begin{aligned}
{\left[\frac{\partial}{\partial t}-\left(\begin{array}{cc}
0 & 1 \\
-A\left(x, D_{x}\right) & 0
\end{array}\right)\right] S(t, s) } & =0 \\
\left.S(\cdot, s)\right|_{t=s} & =0,\left.\quad \frac{\partial S}{\partial t}(\cdot, s)\right|_{t=s}=\mathrm{Id}
\end{aligned}
$$

so that the solution of

$$
\left[\frac{\partial^{2}}{\partial t^{2}}+A\left(x, D_{x}\right)\right] u=f, \quad u(t<0)=0,
$$

is given by

$$
u(y, t)=\int_{0}^{t} \mathrm{P}_{1} S(t, s)\left(\begin{array}{c}
0 \\
f(\cdot, s)
\end{array}\right)(y) \mathrm{d} s=\iint G(y, x, t-s) f(x, s) \mathrm{d} x \mathrm{~d} s,
$$

where we identified the causal Green's function $G(y, x, t-s)$. Here, $\mathrm{P}_{1}$ is the projection, $\mathrm{P}_{1}\left(\begin{array}{c}u \\ \frac{\partial u}{\partial t}\end{array}\right)=u$. Likewise, $S_{+}(t, s)$ solves (for $\left.t \in \mathbb{R}\right)$ the problem

$$
\begin{aligned}
P_{+}\left(x, D_{x}, D_{t}\right) S_{+}(\cdot, s) & =0, \\
\left.S_{+}(\cdot, s)\right|_{t=s} & =\mathrm{Id},
\end{aligned}
$$

so that the causal solution of

$$
P_{+}\left(x, D_{x}, D_{t}\right) u_{+}=f_{+}, \quad f_{+}=\frac{1}{2} \mathrm{i} B\left(x, D_{x}\right)^{-1} f
$$

is given by

$$
u_{+}(y, t)=\int_{-\infty}^{t}\left(S_{+}(t, s) f_{+}(\cdot, s)\right)(y) \mathrm{d} s=\iint G_{+}(y, x, t-s) f_{+}(x, s) \mathrm{d} x \mathrm{~d} s,
$$

while the anticausal solution is given by

$$
u_{+}(y, t)=-\int_{t}^{\infty}\left(S_{+}(t, s) f_{+}(\cdot, s)\right)(y) \mathrm{d} s=\iint G_{+}(y, x, s-t) f_{+}(x, s) \mathrm{d} x \mathrm{~d} s .
$$

A similar construction holds with + replaced by - . 
2.1. Oscillatory integral representation. For sufficiently small $t$ (in the absence of conjugate points), one obtains the oscillatory integral representation,

$$
\left(S_{ \pm}(t) h_{ \pm}\right)(y)=(2 \pi)^{-n} \iint a_{ \pm}(y, t, \xi) \exp \left[\mathrm{i} \phi_{ \pm}(y, t, x, \xi)\right] h_{ \pm}(x) \mathrm{d} x \mathrm{~d} \xi
$$

where

$$
\phi_{ \pm}(y, t, x, \xi)=\alpha_{ \pm}(y, t, \xi)-\langle\xi, x\rangle .
$$

We note that $\alpha_{-}(y, t, \xi)=-\alpha_{+}(y, t,-\xi)$. Singularities are propagated along the bicharacteristics, which are determined by Hamilton's equations generated by the principal symbol $\pm B^{\text {prin }}(x, \xi)$

$$
\frac{\mathrm{d} y^{t}}{\mathrm{~d} t}= \pm \frac{\partial B^{\operatorname{prin}}\left(y^{t}, \eta^{t}\right)}{\partial \eta}, \quad \frac{\mathrm{d} \eta^{t}}{\mathrm{~d} t}=\mp \frac{\partial B^{\operatorname{prin}}\left(y^{t}, \eta^{t}\right)}{\partial y} .
$$

We denote the solution of 2.10 with the $+\operatorname{sign}$ and initial values $(x, \xi)$ at $t=0$ by $\left(y^{t}(x, \xi), \eta^{t}(x, \xi)\right)=\Phi^{t}(x, \xi)$. The solution with the - sign is found upon reversing the time direction and is given by $\left(y^{-t}(x, \xi), \eta^{-t}(x, \xi)\right)$. Away from conjugate points, $y^{t}$ and $\xi$ determine $\eta^{t}$ and $x$; we write $x=\widetilde{x}^{t}(y, \xi)$ and $\eta^{t}=\widetilde{\eta}^{t}(y, \xi)$. (We also use the parametrization in which the roles of $(y, \xi)$ and $(x, \eta)$ are interchanged.) Then

$$
\alpha_{+}(y, t, \xi)=\left\langle\xi, \widetilde{x}^{t}(y, \xi)\right\rangle \text {. }
$$

To highest order,

$$
a_{+}(y, t, \xi)=\left.\left|\frac{\partial\left(y^{t}\right)}{\partial(x)}\right|_{x=\widetilde{x}^{t}(y, \xi), \xi}\right|^{-1 / 2} .
$$

We consider the perturbations of $\left(y^{t}, \eta^{t}\right)$ with respect to the initial conditions $(x, \xi)$,

$$
W^{t}(x, \xi)=\left(\begin{array}{ll}
W_{1}^{t}(x, \xi) & W_{2}^{t}(x, \xi) \\
W_{3}^{t}(x, \xi) & W_{4}^{t}(x, \xi)
\end{array}\right)=\left(\begin{array}{cc}
\partial_{x} y^{t}(x, \xi) & \partial_{\xi} y^{t}(x, \xi) \\
\partial_{x} \eta^{t}(x, \xi) & \partial_{\xi} \eta^{t}(x, \xi)
\end{array}\right)
$$

This matrix solves the (linearized) Hamilton-Jacobi equations,

$$
\frac{\mathrm{d} W^{t}}{\mathrm{~d} t}(x, \xi)=\left(\begin{array}{rr}
\partial_{\eta y} B^{\operatorname{prin}}\left(y^{t}, \eta^{t}\right) & \partial_{\eta \eta} B^{\operatorname{prin}}\left(y^{t}, \eta^{t}\right) \\
-\partial_{y y} B^{\operatorname{prin}}\left(y^{t}, \eta^{t}\right) & -\partial_{y \eta} B^{\operatorname{prin}}\left(y^{t}, \eta^{t}\right)
\end{array}\right) W^{t}(x, \xi),
$$

subject to initial conditions $W^{t=0}=\mathrm{I}$. We note that away from conjugate points, the submatrix $W_{1}^{t}$ is invertible. Because

$$
\widetilde{x}^{t}=\frac{\partial \alpha_{+}}{\partial \xi}, \quad \widetilde{\eta}^{t}=\frac{\partial \alpha_{+}}{\partial y}
$$

integration of 2.13) along $\left(y^{t}, \eta^{t}\right)$ yields:

$$
\begin{aligned}
& \frac{\partial^{2} \alpha_{+}}{\partial y \partial \xi}\left(y^{t}(x, \xi), t, \xi\right)=\left(W_{1}^{t}(x, \xi)\right)^{-1} \\
& \frac{\partial^{2} \alpha_{+}}{\partial \xi^{2}}\left(y^{t}(x, \xi), t, \xi\right)=\left(W_{1}^{t}(x, \xi)\right)^{-1} W_{2}^{t}(x, \xi) \\
& \frac{\partial^{2} \alpha_{+}}{\partial y^{2}}\left(y^{t}(x, \xi), t, \xi\right)=W_{3}^{t}(x, \xi)\left(W_{1}^{t}(x, \xi)\right)^{-1}
\end{aligned}
$$


which we evaluate at $x=\widetilde{x}^{t}(y, \xi)$. It follows that

$$
a_{+}(y, t, \xi)=\left.\left|\operatorname{det} W_{1}^{t}\right|_{x=\widetilde{x}^{t}(y, \xi), \xi}\right|^{-1 / 2} .
$$

The amplitude of $S_{+}(t) \frac{1}{2} \mathrm{i} B^{-1}$, then becomes

$$
a_{+}(y, t, \xi) \frac{1}{2} \mathrm{i} B^{\text {prin }}\left(\widetilde{x}^{t}(y, \xi), \xi\right)^{-1}
$$

to leading order; we denote this amplitude by $\widetilde{a}_{+}(y, t, \xi)$. The amplitude $a_{-}$follows from time reversal: $a_{-}(y, t, \xi)=\overline{a_{+}(y, t,-\xi)}$.

In the case of conjugate points, we use the semigroup property of $S(t, s)$ and decompose the time step into smaller time steps such that in each step the formation of caustics is avoided. Numerically, the size of the smaller time steps can be determined by monitoring the rank-deficiency of $W_{1}^{t}$, see [11] for a more general point of view and Subsection 3.4 for an application.

2.2. The source field. In the absence of caustics, we can change phase variables in the oscillatory integral representation of $G$ according to

$$
\begin{aligned}
G_{+}(y, x, t)= & (2 \pi)^{-1} \iint(2 \pi)^{-n} \int a_{+}\left(y, t^{\prime}, \xi\right) \\
& \exp \left[\mathrm{i} \phi_{+}\left(y, t^{\prime}, x, \xi\right)\right] \mathrm{d} \xi \exp \left[\mathrm{i} \tau\left(t-t^{\prime}\right)\right] \mathrm{d} t^{\prime} \mathrm{d} \tau \\
& =(2 \pi)^{-1} \int a_{+}^{\prime}(y, x, \tau) \exp [\mathrm{i} \tau(t-T(y, x))] \mathrm{d} \tau .
\end{aligned}
$$

By applying the method of stationary phase in the variables $\left(\xi, t^{\prime}\right)$, one can show that the source field can be written in the form 6

$$
G(x, \tilde{x}, t)=(2 \pi)^{-1} \int a^{\prime}(x, \tilde{x}, \tau) \exp [\mathrm{i} \tau(t-T(x, \tilde{x}))] \mathrm{d} \tau .
$$

Here, $\tilde{x}$ is the source location and $T$ is the travel time satisfying the eikonal equation

$$
B^{\text {prin }}\left(x,-\partial_{x} T(x, \tilde{x})\right)=-1
$$

and $a^{\prime}=\mathcal{A}$ to highest order with

$$
|\mathcal{A}(x, \tilde{x}, \tau)|=(2 \pi)^{-(n-1) / 2}\left|\operatorname{det} \frac{\partial(x, \xi, t)}{\partial(y, x, \tau)}\right|^{1 / 2}
$$

see [6] for details. We introduce

$$
n_{\tilde{x}}(x)=\frac{\partial_{x} T(x, \tilde{x})}{\left|\partial_{x} T(x, \tilde{x})\right|}
$$

in view of 2.19,

$$
\left|\partial_{x} T(x, \tilde{x})\right|=\frac{1}{B^{\operatorname{prin}}\left(x, n_{\tilde{x}}(x)\right)} .
$$

We note that through $n_{\tilde{x}}(x)$ we obtain the incidence angle of the source field at $x$. In Section 5.3 we will arrange and study the images with respect to incidence angle. We also note that $n_{\tilde{x}}(x)$ can be estimated from the Poynting vector $-\partial_{t} G(x, \tilde{x}, t) \partial_{x} G(x, \tilde{x}, t)$ at $t=T(x, \tilde{x})$ [35, 36] or from $-\partial_{t} G(x, \tilde{x}, t) \stackrel{(t=0)}{*} \partial_{x} G(x, \tilde{x},-t)$, (possibly normalized by the autocorrelation, $\partial_{t} G(x, \tilde{x}, t) \stackrel{(t=0)}{*} \partial_{t} G(x, \tilde{x},-t)$; note that this normalization is primarily applied to suppress the dependency on $a^{\prime}$ ), for instance in the PDE solution formulation of RTM. 
3. Reverse-time continuation from the boundary. The key results we obtain in this section are the formulation of an oscillatory integral representation and its computation using dyadic parabolic decomposition and wave packets for reversetime continuation with a boundary source. These are also central in the formulation and computation of the inverse scattering and imaging operators presented in Section 4. We introduce Euclidean boundary normal coordinates, $x=\left(x^{\prime}, x_{n}\right)$; that is, $x^{\prime}=\left(x_{1}, \ldots, x_{n-1}\right)$, and $x_{n}=0$ defines the boundary. We let $\Sigma$ denote a bounded open subset of $\left\{(x, t) \in \mathbb{R}_{x}^{n} \times \mathbb{R}_{t} \mid x_{n}=0\right\}$. We denote the restriction to the boundary by $R_{x_{n}}$.

We let $w_{r}$ be an anticausal solution to

$$
\left[\frac{\partial^{2}}{\partial t^{2}}+A\left(x, D_{x}\right)\right] w_{r}(x, t)=\delta\left(x_{n}\right) g\left(x^{\prime}, t\right)
$$

we have $w_{r}=w_{r,+}+w_{r,-}$ with

$$
w_{r,+}(y, t)=-\int_{t}^{\infty}\left(S_{+}(t, s) \frac{1}{2} \mathrm{i} B^{-1} R_{x_{n}}^{*} \widetilde{\Psi}_{\Sigma} g(\cdot, s)\right)(y) \mathrm{d} s
$$

noting that

$$
R_{x_{n}}^{*} g(x, t)=\delta\left(x_{n}\right) g\left(x^{\prime}, t\right)
$$

for any functions $g$ defined on $\mathbb{R}_{x^{\prime}}^{n-1} \times \mathbb{R}_{t}$. Here, $\widetilde{\Psi}_{\Sigma}=\widetilde{\Psi}_{\Sigma}\left(x^{\prime}, t, D_{x^{\prime}}, D_{t}\right)$ is a pseudodifferential cutoff designed to remove grazing rays. The relation between contributions from negative frequencies and positive frequencies is

$$
w_{r,-}(y, t)=\overline{w_{r,+}(y, t)}
$$

We now introduce principal parts of symbols, $C_{ \pm}\left(x^{\prime}, x_{n}, \xi^{\prime}, \tau\right)$, as the solutions for $\zeta$ of

$$
A^{\text {prin }}\left(x^{\prime}, x_{n}, \xi^{\prime}, \zeta\right)=\tau^{2} .
$$

We write $C\left(x^{\prime}, x_{n}, \xi^{\prime}, \tau\right)=C_{+}\left(x^{\prime}, x_{n}, \xi^{\prime}, \tau\right)$. In the further analysis we will need the operator,

$$
C\left(x^{\prime}, x_{n}, D_{t}^{-1} D_{x^{\prime}}, 1\right) \text { at the surface, } x_{n}=0
$$

with principal symbol $C\left(x^{\prime}, x_{n}, \tau^{-1} \xi^{\prime}, 1\right)$.

3.1. Conjugate points. In the case of conjugate points, we introduce a partition of unity into $\Sigma \subset \mathbb{R}_{x^{\prime}}^{n-1} \times \mathbb{R}_{t}$ (with overlap $\delta$ in time). Incorporating this partition of unity in $\widetilde{\Psi}_{\Sigma}$, we obtain a set of cutoffs, $\widetilde{\Psi}_{\Sigma, i j}$. The first index signifies a subdivision in $\mathbb{R}_{x^{\prime}}^{n-1}$ while the second index identifies intervals in time.

To describe the use of the semigroup property, we fix $i$. Let $i=1$ and assume, without loss of generality, that we need two smaller time intervals, $\left[t, t+t_{1}\right]$ and $\left[t+t_{1}, T_{1}\right]$, say, to avoid conjugate points in the smaller time intervals. Then

$$
\begin{gathered}
\int_{t}^{T_{1}}\left(S_{+}(t, s) \frac{1}{2} \mathrm{i} B^{-1} R_{x_{n}}^{*}\left(\widetilde{\Psi}_{\Sigma, 11}+\widetilde{\Psi}_{\Sigma, 12}\right) g(\cdot, s)\right)(y) \mathrm{d} s \\
=\int_{t}^{t+t_{1}}\left(S_{+}(t, s) \frac{1}{2} \mathrm{i} B^{-1} R_{x_{n}}^{*} \widetilde{\Psi}_{\Sigma, 11} g(\cdot, s)\right)(y) \mathrm{d} s \\
+S_{+}\left(t, t+t_{1}-\delta\right) \int_{t+t_{1}-\delta}^{T_{1}}\left(S_{+}\left(t+t_{1}-\delta, s\right) \frac{1}{2} \mathrm{i} B^{-1} R_{x_{n}}^{*} \widetilde{\Psi}_{\Sigma, 12} g(\cdot, s)\right)(y) \mathrm{d} s
\end{gathered}
$$


We now focus on representations for

$$
\begin{aligned}
\int_{t}^{t+t_{1}}\left(S_{+}(t, s) \frac{1}{2} \mathrm{i} B^{-1} R_{x_{n}}^{*} \widetilde{\Psi}_{\Sigma, 11} g(\cdot, s)\right)(y) \mathrm{d} s \text { and } \\
\qquad \int_{t+t_{1}-\delta}^{T_{1}}\left(S_{+}\left(t+t_{1}-\delta, s\right) \frac{1}{2} \mathrm{i} B^{-1} R_{x_{n}}^{*} \widetilde{\Psi}_{\Sigma, 12} g(\cdot, s)\right)(y) \mathrm{d} s,
\end{aligned}
$$

in the absence of conjugate points.

3.2. Oscillatory integral representations. We have

$$
\begin{aligned}
\chi_{n} w_{r,+}(y, t)=(2 \pi)^{-n} \iint_{t}^{\infty} \int \chi_{n} a^{(\mathrm{bkd})}\left(x^{\prime}, s-t, y, \eta\right) \\
\quad \exp \left[\mathrm{i}\left(-\alpha_{+}\left(x^{\prime}, 0, s-t, \eta\right)+\langle\eta, y\rangle\right)\right] g\left(x^{\prime}, s\right) \mathrm{d} x^{\prime} \mathrm{d} s \mathrm{~d} \eta,
\end{aligned}
$$

where

$$
a^{(\mathrm{bkd})}\left(x^{\prime}, s-t, y, \eta\right)=\left.\left|\frac{\partial\left(y^{s-t}\right)}{\partial(x)}\right|_{\eta, x=x^{s-t}\left(x^{\prime}, 0, \eta\right)}\right|^{-1 / 2} \frac{1}{2} \mathrm{i} \tau^{-1} \widetilde{\Psi}_{\Sigma}\left(x^{\prime}, s, \xi^{\prime}, \tau\right)
$$

up to terms of lower order, that is, the error (expressed in $\eta$ ) is of order $\left(1+|\eta|^{2}\right)^{-1}$, and $\chi_{n}$ is a cutoff function which removes contributions for $x_{n}<0$ (the expressions for $\xi^{\prime}$ and $\tau$ in terms of $\eta$ are given in (3.9) and (3.10) below). The operator $\chi_{n} S_{+}(t, s) \frac{1}{2} \mathrm{i} B^{-1} R_{x_{n}}^{*} \widetilde{\Psi}_{\Sigma}$ is a FIO, the canonical relation of which is a subset of

$$
\left\{\left(y, \eta ;\left(y^{s-t}\right)^{\prime}(y, \eta), s-t,\left(\eta^{s-t}\right)^{\prime}(y, \eta),-B^{\text {prin }}(y, \eta)\right) \mid\left(y^{s-t}\right)_{n}(y, \eta)=0\right\} .
$$

The dyadic parabolic decomposition of phase space enters in the reverse-time continuation as

$$
\begin{aligned}
\chi_{n}\left(y_{n}\right) w_{r,+}(y, t)=\chi_{n}\left(y_{n}\right) \sum_{\nu, k} \iint\left\{(2 \pi)^{-n} \int \widehat{\beta}_{\nu, k}(\eta) \widehat{\chi}_{\nu, k}(\eta)\right. \\
\left.a^{(\mathrm{bkd})}\left(x^{\prime}, s-t, y, \eta\right) \exp \left[-\mathrm{i} \alpha_{+}\left(x^{\prime}, 0, s-t, \eta\right)\right] \mathrm{d} \eta\right\} g\left(x^{\prime}, s\right) \mathrm{d} x^{\prime} \mathrm{d} s .
\end{aligned}
$$

Fixing $(\nu, k)$ corresponds with (directional) "controlled illumination."

3.3. Boundary source decomposition; wave packets in space-time. We change phase variables in the representation for $w_{r,+}$. We could do this in two steps, changing parametrizations from $\left(\left(x^{\prime}, x_{n}\right), \eta\right)$ to $\left(y,\left(\xi^{\prime}, \xi_{n}\right)\right)$ and then $\left(s, \xi_{n}\right)$ to $\left(x_{n}, \tau\right)$. Here, we carry out this change in a single step:

$$
\begin{aligned}
& \chi_{n} w_{r,+}(y, t)=(2 \pi)^{-2 n} \iiint_{t}^{\infty} \iint a^{(\mathrm{bkd})}\left(x^{\prime}, s-t, y, \eta\right) \\
& \exp \left[\mathrm{i}\left(-\alpha_{+}\left(x^{\prime}, 0, s-t, \eta\right)+\langle\eta, y\rangle\right)\right] \exp \left[\mathrm{i}\left(\tau s+\left\langle\xi^{\prime}, x^{\prime}\right\rangle\right)\right] \mathrm{d} \eta \mathrm{d} x^{\prime} \mathrm{d} s \widehat{g}\left(\xi^{\prime}, \tau\right) \mathrm{d} \xi^{\prime} \mathrm{d} \tau
\end{aligned}
$$

applying the method of stationary phase in $\left(\eta, x^{\prime}, s\right)$ yields solving

$$
\begin{aligned}
\partial_{\eta} \alpha_{+}\left(x^{\prime}, 0, s-t, \eta\right) & =y, \\
\partial_{x^{\prime}} \alpha_{+}\left(x^{\prime}, 0, s-t, \eta\right) & =\xi^{\prime}, \\
\partial_{s} \alpha_{+}\left(x^{\prime}, 0, s-t, \eta\right) & =\tau
\end{aligned}
$$


for given $\left(y, \xi^{\prime}, \tau\right)$ and $t$ fixed (which is viewed as a parameter here). The solutions, $\left(\eta_{0}, x_{0}^{\prime}, s_{0}\right)$, are the stationary points of $-\alpha_{+}\left(x^{\prime}, 0, s-t, \eta\right)+\langle\eta, y\rangle+\tau s+\left\langle\xi^{\prime}, x^{\prime}\right\rangle$. We have $s_{0}>t$. These equations imply that

$$
\left.\begin{array}{rl}
y & =\widetilde{y}^{s_{0}-t}\left(x_{0}^{\prime}, 0, \eta_{0}\right) \\
\xi^{\prime} & =\widetilde{\xi}^{s_{0}-t \prime}\left(x_{0}^{\prime}, 0, \eta_{0}\right)
\end{array}\right\} \text { that is, }\left(x_{0}^{\prime}, 0, \widetilde{\xi}^{s_{0}-t \prime}, C\left(x_{0}^{\prime}, 0, \widetilde{\xi}^{s_{0}-t \prime}, \tau\right)\right) \stackrel{\Phi^{s_{0}-t}}{\rightarrow}\left(\widetilde{y}^{s_{0}-t}, \eta_{0}\right) \text {. }
$$

For given $t, s_{0}$ is determined since $\left(x_{0}^{\prime}, 0\right), \eta_{0}$ and $s_{0}-t$ determine a unique ray, in view of the absence of conjugate points. Thus we need to solve

$$
\begin{aligned}
\eta_{0} & =\widetilde{\eta}^{s_{0}-t}\left(y, \xi^{\prime}, C\left(x_{0}^{\prime}, 0, \xi^{\prime}, \tau\right)\right), \\
x_{0}^{\prime} & =\widetilde{x}^{s_{0}-t}\left(y, \xi^{\prime}, C\left(x_{0}^{\prime}, 0, \xi^{\prime}, \tau\right)\right), \\
0 & =\widetilde{x}_{n}^{s_{0}-t}\left(y, \xi^{\prime}, C\left(x_{0}^{\prime}, 0, \xi^{\prime}, \tau\right)\right) \text { or } s_{0}=T\left(x_{0}^{\prime}, 0, y\right)+t,
\end{aligned}
$$

for $\left(\eta_{0}, x_{0}^{\prime}, s_{0}\right)$. To obtain a unique solution, in general, we need to localize $g$, which we do by substituting a wave packet contribution, that is, $g_{\gamma} \widehat{\varphi}_{\gamma}\left(\xi^{\prime}, \tau\right)$ for $\widehat{g}\left(\xi^{\prime}, \tau\right)$. Then

$$
\begin{aligned}
-\alpha_{+}\left(x_{0}^{\prime}\left(y, \xi^{\prime}, \tau ; t\right), 0, s_{0}\left(y, \xi^{\prime}, \tau ; t\right)-t, \eta_{0}\left(y, \xi^{\prime}, \tau ; t\right)\right) & +\left\langle\eta_{0}\left(y, \xi^{\prime}, \tau ; t\right), y\right\rangle \\
=-\left\langle\eta_{0}\left(y, \xi^{\prime}, \tau ; t\right), y^{s_{0}\left(y, \xi^{\prime}, \tau ; t\right)-t}\left(x_{0}^{\prime}\left(y, \xi^{\prime}, \tau ; t\right), 0,\right.\right. & \left.\left.\eta_{0}\left(y, \xi^{\prime}, \tau ; t\right)\right)\right\rangle \\
& +\left\langle\eta_{0}\left(y, \xi^{\prime}, \tau ; t\right), y\right\rangle=0
\end{aligned}
$$

while

$$
\begin{aligned}
\tau & s_{0}\left(y, \xi^{\prime}, \tau ; t\right)+\left\langle\xi^{\prime}, x_{0}^{\prime}\left(y, \xi^{\prime}, \tau ; t\right)\right\rangle \\
=\tau s_{0}\left(y, \xi^{\prime}, \tau ; t\right)+\left\langle\xi^{\prime}, \widetilde{x}^{s_{0}\left(y, \xi^{\prime}, \tau ; t\right)-t^{\prime}}\left(y, \xi^{\prime}, C\left(x_{0}^{\prime}\left(y, \xi^{\prime}, \tau ; t\right), 0, \xi^{\prime}, \tau\right)\right)\right\rangle & =: \theta_{+}\left(y, t, \xi^{\prime}, \tau\right) .
\end{aligned}
$$

We evaluate

$$
\begin{gathered}
\left.\frac{\partial^{2}\left[-\alpha_{+}\left(x^{\prime}, 0, s-t, \eta\right)+\langle\eta, y\rangle+\tau s+\left\langle\xi^{\prime}, x^{\prime}\right\rangle\right]}{\partial \eta \partial \eta}\right|_{\left(\eta_{0}, x_{0}^{\prime}, s_{0}\right)} \\
=-\left.\frac{\partial^{2}\left[\alpha_{+}\left(x^{\prime}, 0, s-t, \eta\right)\right]}{\partial \eta \partial \eta}\right|_{\left(\eta_{0}, x_{0}^{\prime}, s_{0}\right)}=-\left(W_{1}^{s_{0}-t}\left(y, \eta_{0}\right)\right)^{-1} W_{2}^{s_{0}-t}\left(y, \eta_{0}\right), \\
\left.\frac{\partial^{2}\left[-\alpha_{+}\left(x^{\prime}, 0, s-t, \eta\right)+\langle\eta, y\rangle+\tau s+\left\langle\xi^{\prime}, x^{\prime}\right\rangle\right]}{\partial \eta \partial x^{\prime}}\right|_{\left(\eta_{0}, x_{0}^{\prime}, s_{0}\right)} \\
=-\left.\frac{\partial^{2} \alpha_{+}\left(x^{\prime}, 0, s-t, \eta\right)}{\partial \eta \partial x^{\prime}}\right|_{\left(\eta_{0}, x_{0}^{\prime}, s_{0}\right)}=-\left[\left(W_{1}^{s_{0}-t}\left(y, \eta_{0}\right)\right)^{-1}\right]^{\prime},
\end{gathered}
$$

and

$$
\begin{aligned}
& \left.\frac{\partial^{2}\left[-\alpha_{+}\left(x^{\prime}, 0, s-t, \eta\right)+\langle\eta, y\rangle+\tau s+\left\langle\xi^{\prime}, x^{\prime}\right\rangle\right]}{\partial x^{\prime} \partial x^{\prime}}\right|_{\left(\eta_{0}, x_{0}^{\prime}, s_{0}\right)} \\
& =-\left.\frac{\partial^{2} \alpha_{+}\left(x^{\prime}, 0, s-t, \eta\right)}{\partial x^{\prime} \partial x^{\prime}}\right|_{\left(\eta_{0}, x_{0}^{\prime}, s_{0}\right)}=-{ }^{\prime}\left[W_{3}^{s_{0}-t}\left(y, \eta_{0}\right)\right]\left[\left(W_{1}^{s_{0}-t}\left(y, \eta_{0}\right)\right)^{-1}\right]^{\prime},
\end{aligned}
$$


subject to the substitutions according to (3.11)-(3.13), and then

$$
\begin{gathered}
\left.\left.\frac{\partial^{2}\left[-\alpha_{+}\left(x^{\prime}, 0, s-t, \eta\right)+\langle\eta, y\rangle+\tau s+\left\langle\xi^{\prime}, x^{\prime}\right\rangle\right]}{\partial s^{2}}\right|_{\left(\eta_{0}, x_{0}^{\prime}, s_{0}\right)}\right|_{\left(\eta_{0}, x_{0}^{\prime}, s_{0}\right)}=-\left.\frac{\partial \tau}{\partial s}\right|_{s=s_{0}-t}, \\
\left.\frac{\partial^{2}\left[-\alpha_{+}\left(x^{\prime}, 0, s-t, \eta\right)+\langle\eta, y\rangle+\tau s+\left\langle\xi^{\prime}, x^{\prime}\right\rangle\right]}{\partial s \partial \eta}\right|_{\left(\eta_{0}, x_{0}^{\prime}, s_{0}\right)}, \\
=-\left.\frac{\partial^{2} \alpha_{+}\left(x^{\prime}, 0, s-t, \eta\right)}{\partial s \partial \eta}\right|_{\left(\eta_{0}, x_{0}^{\prime}, s_{0}\right)}=-\left.\frac{\partial \widetilde{y}^{s-t}}{\partial s}\right|_{s=s_{0}}\left(x_{0}^{\prime}, 0, \eta_{0}\right),
\end{gathered}
$$

and

$$
\begin{aligned}
\left.\frac{\partial^{2}\left[-\alpha_{+}\left(x^{\prime}, 0, s-t, \eta\right)+\langle\eta, y\rangle+\tau s+\left\langle\xi^{\prime}, x^{\prime}\right\rangle\right]}{\partial s \partial x^{\prime}}\right|_{\left(\eta_{0}, x_{0}^{\prime}, s_{0}\right)} \\
=-\left.\frac{\partial^{2} \alpha_{+}\left(x^{\prime}, 0, s-t, \eta\right)}{\partial s \partial x^{\prime}}\right|_{\left(\eta_{0}, x_{0}^{\prime}, s_{0}\right)}=-\left.\frac{\partial \widetilde{\xi}^{s-t}}{\partial s}\right|_{s=s_{0}}\left(x_{0}^{\prime}, 0, \eta_{0}\right) .
\end{aligned}
$$

From these expressions we form the Hessian which is used to transform $a^{(\mathrm{bkd})}\left(x^{\prime}, s-\right.$ $t, y, \eta)$ to the amplitude $a_{b}^{(\mathrm{bkd})}\left(y, t, \xi^{\prime}, \tau\right)$, so that

$$
\begin{aligned}
\chi_{n} w_{r,+}(y, t)=(2 \pi)^{-n} \sum_{\gamma} g_{\Sigma, \gamma} \iint \chi_{n} a_{b}^{(\mathrm{bkd})}\left(y, t, \xi^{\prime}, \tau\right) & \\
& \exp \left[\mathrm{i} \theta_{+}\left(y, t, \xi^{\prime}, \tau\right)\right] \widehat{\varphi}_{\gamma}\left(\xi^{\prime}, \tau\right) \mathrm{d} \xi^{\prime} \mathrm{d} \tau .
\end{aligned}
$$

Essentially, this representation corresponds with local coordinates $\left(x_{n}, y, \xi^{\prime}, \tau\right)$ for the canonical relation of the solution operator with $t$ fixed.

3.4. Algorithm. We adapt the "box algorithm" for the multiscale discrete approximation of FIOs developed in [2] to (3.22), with accuracy $\mathcal{O}\left(2^{-k / 2}\right)$ at frequency scale $k$. In the general case, the medium can be laterally varying at the boundary. Then we need to employ compactly supported cutoff functions in $x^{\prime}$, realized by the partition of unity $\widetilde{\Psi}_{\Sigma, i j}$. Within each cutoff $i$ the lateral variation of the (smooth) velocity model is assumed to be negligible at the boundary, and the algorithm outlined below can then be applied for each cutoff $i$ separately.

For convenience of notation, we now assume that the wave speed does not vary laterally at the surface and fix $i=1$. Without loss of generality, we suppose that we need $N_{s}$ time intervals $\left[t+\left(n_{s}-1\right) t_{1}, t+n_{s} t_{1}\right], n_{s}=1, \ldots, N_{s}$, of duration $t_{1}=\left(T_{1}-t\right) / N_{s}$ in order to avoid the formation of conjugate points. Numerically, such a covering of sub-time intervals can be determined straightforwardly from the points of rank-deficiency of the matrix $W_{1}^{t}$ of the Hamiltonian system as detailed in 11] and sketched below.

Let us consider one frequency box $\hat{\chi}_{\nu, k}$. We begin with computing the bicharacteristics (rays) of the Hamiltonian system, $\left(x_{0}^{\prime}, 0, \nu\right) \mapsto(y, \eta)=\left(\tilde{y}^{s}, \tilde{\eta}^{s}\right)$, i.e. $\left(x_{0}^{\prime}, 0, \nu\right)=$ 
$\frac{\partial \theta_{+}\left(y, t, \xi^{\prime}, \tau\right)}{\partial\left(\xi^{\prime}, \tau\right)}$ where $s \in\left(0, t_{1}\right]$. For each time interval $n_{s}$, we thus obtain the coordinate transform [2]

$$
T_{\nu, k}^{\left(n_{s}\right)}(y)=\left(x_{0}^{\prime}, s+t+\left(n_{s}-1\right) t_{1}\right) .
$$

The solution of the corresponding Hamilton-Jacobi system yields the propagator matrix $W^{s}$ from which we obtain the quantities

$$
\begin{aligned}
& \frac{\partial^{2} \theta_{+}\left(y, t, \xi^{\prime}, \tau\right)}{\partial y \partial\left(\xi^{\prime}, \tau\right)}=\left(W_{1}^{s}\right)^{-1} \\
& \frac{\partial^{2} \theta_{+}\left(y, t, \xi^{\prime}, \tau\right)}{\partial^{2}\left(\xi^{\prime}, \tau\right)}=-\left(W_{1}^{s}\right)^{-1} W_{2}^{s} \\
& \frac{\partial^{2} \theta_{+}\left(y, t, \xi^{\prime}, \tau\right)}{\partial^{2} y}=W_{3}^{s}\left(W_{1}^{s}\right)^{-1} .
\end{aligned}
$$

We can now apply the box algorithm to each time interval $n_{s}$ and obtain the (partially) reverse-time continued wave field from (data) boundary sources

$$
\begin{aligned}
& w_{r,+}^{\left(n_{s}\right)}\left(y, t+\left(n_{s}-1\right) t_{1}\right)=\sum_{\nu, k} a^{(\mathrm{bkd})}(y, \nu) \sum_{r=1}^{R_{\nu, k}} \alpha_{\nu, k}^{(r)}(y) \\
& \sum_{\left(\xi^{\prime}, \tau\right)} e^{\mathrm{i}\left\langle T_{\nu, k}^{\left(n_{s}\right)}(y),\left(\xi^{\prime}, \tau\right)\right\rangle} \hat{g}\left(\xi^{\prime}, \tau\right) \hat{\beta}_{\nu, k}\left(\xi^{\prime}, \tau\right) \hat{\chi}_{\nu, k}\left(\xi^{\prime}, \tau\right) \hat{\vartheta}_{\nu, k}^{(r)}\left(\xi^{\prime}, \tau\right)
\end{aligned}
$$

where $\alpha_{\nu, k}^{(r)}$ and $\vartheta_{\nu, k}^{(r)}$ are the expansion functions arising in the tensor-product representation of the complex exponential of the second-order Taylor expansion term of $\theta_{+}$ on the frequency box $\hat{\chi}_{\nu, k}[2]$.

ALGORITHM 1: Outline of reverse-time continuation from the boundary in the case of conjugate points. In the absence of caustics, the algorithm reduces to Part I, with $N_{s}=1$.

PART I - REVERSE-TIME CONTINUATION FROM THE BOUNDARY, SEMIGROUP 1

for $n_{s}=1: N_{s}$ end

1. compute coordinate transforms $T_{\nu, k}$ and propagator matrices $W$

2. compute $w_{r,+}^{\left(n_{s}\right)}\left(y, t+\left(n_{s}-1\right) t_{1}\right)$ : box algorithm, 3.23

Part II - halF WAVE EQuation REVERSE-Time CONTINUATion, SEMigroups

for $n_{p}=2: N_{s}$

for $n_{s}=n_{p}: N_{s}$

half wave equation evolution operator $P_{t_{1}}$ : box algorithm

end $w_{r,+}^{\left(n_{s}\right)}\left(y, t+\left(n_{s}-n_{p}\right) t_{1}\right)=P_{t_{1}} w_{r,+}^{\left(n_{s}\right)}\left(y, t+\left(n_{s}-n_{p}+1\right) t_{1}\right)$

end

$\underline{\text { WAVE FIELD } \quad w_{r,+}(y, t)=\sum_{n_{s}=1}^{N_{s}} w_{r,+}^{\left(n_{s}\right)}(y, t)}$

To obtain the final reverse-time continued wave fields $w_{r,+}^{\left(n_{s}\right)}(y, t)$, we construct a parametrix for the Cauchy initial value problems for the half wave equation with initial data $w_{r,+}^{\left(n_{s}\right)}\left(y, t+\left(n_{s}-1\right) t_{1}\right), n_{s}=2, \ldots, N_{s}$, initial time $t+\left(n_{s}-1\right) t_{1}$ and final time $t$. We compute these parametrices using the box algorithm (this has been studied 
in detail in [2]). We make use of the semigroup property and obtain the parametrix for the reverse-time interval $\left[t+\left(n_{s}-1\right) t_{1}, t\right]$ as the composition of the parametrices for the time intervals $\left[t+\left(n_{s}-n_{p}+1\right) t_{1}, t+\left(n_{s}-n_{p}\right) t_{1}\right], n_{p}=2, \ldots, n_{s}$. Finally, we have

$$
w_{r,+}(y, t)=\sum_{n_{s}=1}^{N_{s}} w_{r,+}^{\left(n_{s}\right)}(y, t)
$$

The different steps involved in modeling receiver wave propagation from the boundary in reverse-time are summarized in Algo. 1 and illustrated in Fig. 2 for a numerical example that is detailed in Section 5 .

The coordinate transform $T_{\nu, k}^{\left(n_{s}\right)}(y)$ and the propagator matrix $W^{s}$ can numerically be evaluated as follows. For simplicity, we consider the case of isotropic medium. Let $c$ be the wave speed at the boundary and $\nu=\left(\nu^{\prime}, \nu_{n}\right)=\left(\xi^{\prime}, \tau\right) /\left|\left(\xi^{\prime}, \tau\right)\right|$. Then $T_{\nu, k}^{\left(n_{s}\right)}(y)$ follows from the bicharacteristics (rays) of the half wave equation supplemented with initial conditions $y^{0}=\left(x^{\prime}, 0\right), \eta^{0}=\left(\eta^{0^{\prime}}, \eta_{n}^{0}\right)=\frac{c}{\nu_{n}}\left(\nu^{\prime}, \sqrt{\nu_{n}^{2} / c^{2}-\left|\nu^{\prime}\right|^{2}}\right.$ ) (for evolution time s). Similarly, $W^{s}$ is obtained as the solution of the Hamilton-Jacobi system associated with the half wave equation with initial conditions $W_{2}^{0}=W_{3}^{0} \equiv 0, W_{1}^{0}=$ $\left(\begin{array}{cc}\mathbf{I}_{n-1} & 0 \\ c \eta^{\prime} & c \eta_{n}\end{array}\right)$ and $W_{4}^{0}=\frac{c}{\nu_{n}}\left(\begin{array}{cc}\mathbf{I}_{n-1} & \frac{c \nu^{\prime}}{\nu_{n} \eta_{n}} \\ \frac{\nu^{\prime}}{\nu_{n}} & \frac{c\left|\nu^{\prime}\right|^{2}}{\nu_{n}^{2} \eta_{n}}\end{array}\right)$.

Finally, the duration $t_{1}$ for the time intervals is fixed numerically to be smaller than the length of the largest time interval $\left(0, t^{*}\right]$ for which $W_{1}^{s}, s \in\left(0, t^{*}\right]$, is nonsingular for the discrete set of values for $\nu$ considered. Note that if conjugate points are detected in the subsequent time-stepping intervals, the concerned time intervals can be further broken up into intervals of smaller size, again using the semigroup property, without the need to recompute the reverse-time continuation up to these points.

4. Inverse scattering. We assume that a source at $\tilde{x}$ generates the data, $d_{\Sigma}\left(x^{\prime}, t\right)$. We introduce the pseudodifferential operator [6]

$$
\mathcal{N}\left(x^{\prime}, D_{x^{\prime}}, D_{t}\right)=-2 \mathrm{i} D_{t} \frac{\partial B^{\text {prin }}}{\partial \xi_{n}}\left(x^{\prime}, 0, D_{t}^{-1} D_{x^{\prime}}, C\left(x^{\prime}, 0, D_{t}^{-1} D_{x^{\prime}}, 1\right)\right) .
$$

Furthermore, we introduce the pseudodifferential cutoff, $\Psi_{\Sigma}$, which acts as a smooth cutoff which goes to zero near $\partial \Sigma$, removes direct rays, and removes grazing rays; that is, its symbol vanishes where

$$
\frac{\partial B^{\text {prin }}}{\partial \xi_{n}}\left(x^{\prime}, 0, \tau^{-1} \xi^{\prime}, C^{\text {prin }}\left(0, x^{\prime}, \tau^{-1} \xi^{\prime}, 1\right)\right)=0 .
$$

These cutoffs commute up to leading order (through the product of their symbols), $\widetilde{\Psi}_{\Sigma} \Psi_{\Sigma}=\Psi_{\Sigma} \widetilde{\Psi}_{\Sigma}$, which follows from the standard calculus of pseudodifferential operators [30].

We let $w_{r}$ be an anticausal solution of (3.1) with

$$
g\left(x^{\prime}, t\right)=\mathcal{N}\left(x^{\prime}, D_{x^{\prime}}, D_{t}\right) \Psi_{\Sigma}\left(x^{\prime}, t, D_{x^{\prime}}, D_{t}\right) d_{\Sigma}\left(x^{\prime}, t\right) .
$$

We define first-order partial differential and pseudodifferential operators $\Xi\left(x, D_{x}, D_{t}\right)$ and $\Theta\left(x, D_{x}, D_{t}\right)$ with (principal) symbols

$$
\begin{aligned}
& \Xi_{0}(x, \xi, \tau)=\tau, \quad \Xi_{j}(x, \xi, \tau)=\xi_{j} \\
& \Theta_{0}(x, \xi, \tau)=\tau, \quad \Theta_{j}(x, \xi, \tau)=\tau \frac{\partial B^{\text {prin }}}{\partial \xi_{j}}(x, \xi) .
\end{aligned}
$$


We then define the pseudodifferential operator $L$ and the operator $K$ as

$$
\begin{aligned}
L w(y, t) & =\mathcal{A}\left(y, \tilde{x}, D_{t}\right)^{-1} 2 \mathrm{i} D_{t} \sum_{p=0}^{n} \Xi_{p}\left(y,-\partial_{y} T(y, \tilde{x}), 1\right) \Theta_{p}\left(y, D_{y}, D_{t}\right) w(y, t), \\
K w(y) & =w(y, T(y, \tilde{x})) .
\end{aligned}
$$

Operator $K$ is a restriction to a hypersurface in $\mathbb{R}^{n+1}$. The imaging operator, $H$, is then defined as

$$
H d_{\Sigma}(y)=\left(K L\left(w_{r,+}+w_{r,-}\right)\right)(y) .
$$

To leading order symbols, we get

$$
\begin{aligned}
& L \chi_{n} w_{r,+}(y, t)=\frac{1}{(2 \pi)^{n}} \mathcal{A}\left(y, \tilde{x}, D_{t}\right)^{-1} 2 \mathrm{i} D_{t} \iint \chi_{n} a_{b}^{(\mathrm{bkd})}\left(y, t, \xi^{\prime}, \tau\right) \\
& \times \sum_{p=0}^{n} \Xi_{p}\left(y,-\partial_{y} T(y, \tilde{x}), 1\right) \Theta_{p}\left(y, \partial_{y} \theta_{+}, \partial_{t} \theta_{+}\right) w(y, T(y, \tilde{x})) \\
& \times \exp \left[\mathrm{i} \theta_{+}\left(y, t, \xi^{\prime}, \tau\right)\right] \widehat{g}\left(\xi^{\prime}, \tau\right) \mathrm{d} \xi^{\prime} \mathrm{d} \tau .
\end{aligned}
$$

4.1. Isotropic case. In the isotropic case,

$$
\begin{aligned}
A^{\text {prin }}(x, \xi) & =c(x)^{2} \xi^{2}, \\
B^{\text {prin }}(x, \xi) & =c(x)|\xi|, \\
C\left(x^{\prime}, 0, \tau^{-1} \xi^{\prime}, 1\right) & =\sqrt{1-c\left(x^{\prime}, 0\right)^{2} \tau^{-2} \xi^{\prime 2}}, \\
\Theta_{0}(x, \xi, \tau) & =\tau, \quad \Theta_{j}(x, \xi, \tau)=\tau c(x) \frac{\xi_{j}}{|\xi|}
\end{aligned}
$$

and (4.4) simplifies to

$$
\begin{aligned}
& L \chi_{n} w_{r,+}(y, t)=\frac{1}{(2 \pi)^{n}} \frac{1}{\mathcal{A}_{\mathrm{g}}(y, \tilde{x})} \partial_{t}^{-\frac{n+1}{2}} \int(\mathrm{i} \tau)^{(n-3) / 2} \int \chi_{n} a_{b}^{(\mathrm{bkd})}\left(y, t, \xi^{\prime}, \tau\right) \\
& \times \mathrm{i}\left[\partial_{t} \theta_{+}\left(y, t, \xi^{\prime}, \tau\right)+c(y) n_{\tilde{x}}(y) \cdot \partial_{y} \theta_{+}\left(y, t, \xi^{\prime}, \tau\right)\right] \\
& \times \exp \left[\mathrm{i} \theta_{+}\left(y, t, \xi^{\prime}, \tau\right)\right] \widehat{g}\left(\xi^{\prime}, \tau\right) \mathrm{d} \xi^{\prime} \mathrm{d} \tau
\end{aligned}
$$

using that

$$
\mathcal{A}(y, \tilde{x}, \tau)=\mathcal{A}_{\mathrm{g}}(y, \tilde{x})(\mathrm{i} \tau)^{(n-3) / 2} .
$$

Operator $\partial_{t}^{-\frac{n+1}{2}}$ is to be read as the pseudodifferential operator with symbol $\tau \mapsto$ $\tilde{\sigma}(\tau)(\mathrm{i} \tau)^{-\frac{n+1}{2}}$ in which $\tilde{\sigma}$ is a smooth function, valued 1 except for the origin where it is 0 .

4.2. Computation. We can use $(3.2)$ in the computations. Through a simple modification, we can incorporate the imaging condition in the box algorithm for reverse-time continuation from the boundary detailed in Section 3.4 yielding an RTM imaging algorithm. Without loss of generality, we assume here that the source signature is a delta function; general discrete source signatures can be accommodated 
ALGORITHM 2: Outline of inverse scattering in the case of conjugate points. In the absence of caustics, the algorithm reduces to Part I, with $N_{s}=1$.

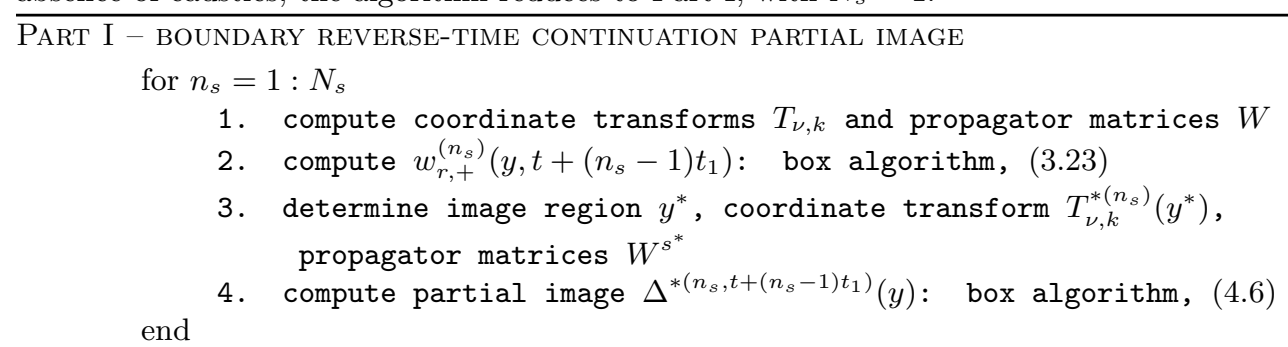

PART II - HALF WAVE EQUATION REVERSE-TIME CONTINUATION PARTiAL IMAGE

for $n_{p}=2: N_{s}$

for $n_{s}=n_{p}: N_{s}$

1. half wave equation evolution operator $P_{t_{1}}$ : box algorithm

$w_{r,+}^{\left(n_{s}\right)}\left(y, t+\left(n_{s}-n_{p}\right) t_{1}\right)=P_{t_{1}} w_{r,+}^{\left(n_{s}\right)}\left(y, t+\left(n_{s}-n_{p}+1\right) t_{1}\right)$

2. image region $\tilde{y}^{s *}: s^{*}=t+\left(n_{s}-n_{p}+1\right) t_{1}-T\left(\tilde{y}^{s *}, \tilde{x}\right)$

3. coordinate transform, propagator matrices

$\tilde{T}_{\nu, k}^{*\left(n_{s}\right)}\left(\tilde{y}^{s *}\right)=y_{0}, \tilde{W}^{*}=W\left(y_{0}, \eta_{0}, s^{*}\right)$

4. evaluate partial image $\tilde{\Delta}^{*\left(n_{s}, n_{p}\right)}(y)$ : box algorithm end

end

IMAGE $\Delta_{d_{\Sigma}}(y)=\sum_{n_{s}=1}^{N_{s}} \Delta^{*\left(n_{s}, t+\left(n_{s}-1\right) t_{1}\right)}(y)+\sum_{n_{p}=2}^{N_{s}} \sum_{n_{s}=n_{p}}^{N_{s}} \tilde{\Delta}^{*\left(n_{s}, n_{p}\right)}(y)$

for in a straight-forward way by viewing them as a weighted sum of delta functions shifted by the time step for solving the Hamilton-Jacobi equations. Suppose that the source travel time $T(y, \tilde{x})$ and amplitude $\mathcal{A}(y, \tilde{x}, \tau)$ have been evaluated for the image region (here, by evaluating the corresponding Hamiltonian and Hamilton-Jacobi system, i.e. ray-tracing; cf. Section 2.2. We begin with the evaluation of the imaging operator $H$ for partial reverse-time continuation from the boundary (cf. Algo. 1. Part I). We obtain a contribution of time interval $n_{s}$ to the image at $y^{*}=y^{*}\left(x^{\prime}, 0, s^{*}\right)$ if $\left(n_{s}-1\right) t_{1}+t \leq T\left(y^{*}, \tilde{x}\right) \leq n_{s} t_{1}+t-s^{*}$. Subject to this condition, the coordinate transform for image point $y^{*}$ is given by $T_{\nu, k}^{*\left(n_{s}\right)}\left(y^{*}\right)=\left(x_{0}^{\prime}, T\left(y^{*}, \tilde{x}\right)+s^{*}\right)$ and the propagator matrices are given by $W^{s^{*}}$. Application of the box algorithm with $T_{\nu, k}^{*\left(n_{s}\right)}$ and $W^{s^{*}}$ with $\mathcal{A}$ incorporated in the amplitude $\tilde{a}^{*}$ yields the partial image

$$
\begin{aligned}
\Delta^{*\left(n_{s}, t+\left(n_{s}-1\right) t_{1}\right)}(y) & =\sum_{\nu, k} \tilde{a}^{*}(y, \nu) \sum_{r=1}^{R_{\nu, k}} \alpha_{\nu, k}^{*(r)}(y) \\
& \times \sum_{\left(\xi^{\prime}, \tau\right)} e^{\mathrm{i}\left\langle T_{\nu, k}^{*\left(n_{s}\right)}(y),\left(\xi^{\prime}, \tau\right)\right\rangle} \hat{g}\left(\xi^{\prime}, \tau\right) \hat{\beta}_{\nu, k}\left(\xi^{\prime}, \tau\right) \hat{\chi}_{\nu, k}\left(\xi^{\prime}, \tau\right) \hat{\vartheta}_{\nu, k}^{*(r)}\left(\xi^{\prime}, \tau\right) .
\end{aligned}
$$

In the case of conjugate points $\left(n_{s}>1\right)$, we proceed with the evaluation of $H$ for the subsequent half wave equation reverse-time continuation of the wave fields $w_{r,+}^{\left(n_{s}\right)}(y, t+$ $\left.\left(n_{s}-1\right) t_{1}\right)$ (cf. Algo. 1 1 Part II). Consider continuation of $w_{r,+}^{\left(n_{s}\right)}\left(y, t+\left(n_{s}-1\right) t_{1}\right)$ to $w_{r,+}^{\left(n_{s}\right)}\left(y, t+\left(n_{s}-2\right) t_{1}\right)\left(n_{p}=2\right)$. In this process, we compute the bicharacteristics $\left(\tilde{y}^{s}\left(y_{0}, \eta_{0}\right), \tilde{\eta}^{s}\left(y_{0}, \eta_{0}\right)\right)$ for $s \in\left(0, t_{1}\right]$. We can easily monitor the condition $s^{*}=t+$ 

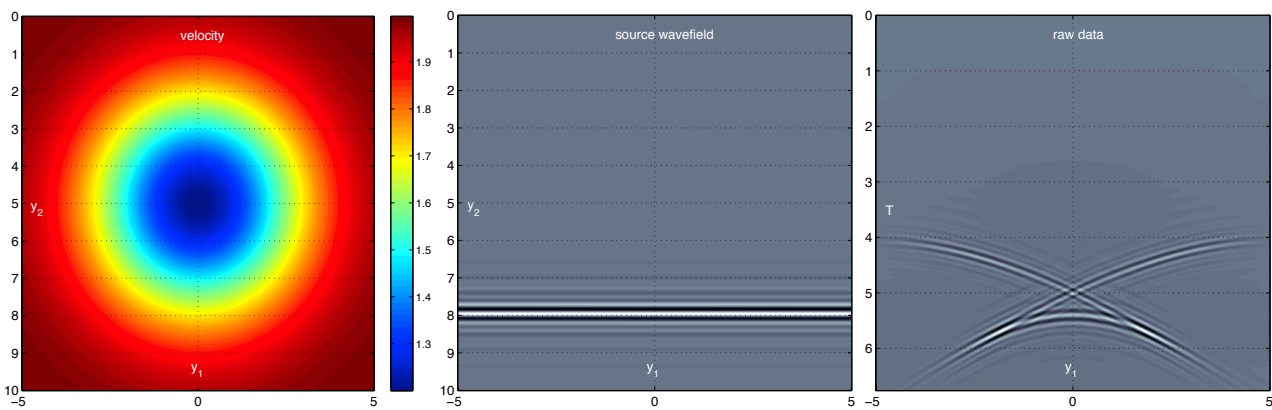

FIG. 1. Reverse-time continuation from a boundary: Velocity model (left), initial wave field at $t=0$ (center) and data collected at the boundary $y_{2}=0$ (right).

$\left(n_{s}-1\right) t_{1}-T\left(\tilde{y}^{s *}, \tilde{x}\right)$ under which we obtain a contribution to the image at $\tilde{y}^{s *}$. The associated coordinate transform is given by $\tilde{T}_{\nu, k}^{*\left(n_{s}\right)}\left(\tilde{y}^{s *}\right)=y_{0}$, and the propagator matrices by $\tilde{W}^{*}=W\left(y_{0}, \eta_{0}, s^{*}\right)$. With these ingredients, application of the box algorithm yields the partial image $\tilde{\Delta}^{*\left(n_{s}, n_{p}\right)}(y)$; we obtain the final image

$$
\Delta_{d_{\Sigma}}(y)=\sum_{n_{s}=1}^{N_{s}} \Delta^{*\left(n_{s}, t+\left(n_{s}-1\right) t_{1}\right)}(y)+\sum_{n_{p}=2}^{N_{s}} \sum_{n_{s}=n_{p}}^{N_{s}} \tilde{\Delta}^{*\left(n_{s}, n_{p}\right)}(y) .
$$

The structure of the inverse scattering procedure is summarized in Algo. 2. Note that in the evaluation of the partial images $\Delta^{*}$ and $\tilde{\Delta}^{*}$, we can gather the incident angles $\eta\left(y^{*} ; \nu, k ; n_{s}, n_{p}\right)$ of the reverse-time continued wave field, which we can, for instance, use for monitoring scattering angles as illustrated in Section 5.3 .

5. Numerical examples. We illustrate the performance of our algorithm in two applications: Reverse-time continuation from the boundary of an upgoing wave field in the presence of conjugate points and imaging of conormal singularities using reverse-time continuation of boundary reflection data. We consider the isotropic case. Although applicable in general dimension, we restrict ourselves here to dimension 2. The sources in these examples, $g\left(x^{\prime}, t\right)$ and $d_{\Sigma}\left(x^{\prime}, t\right)$, respectively, are generated using a time domain finite difference method. The computational domain is of size $N \times N=512 \times 512$.

5.1. Reverse-time continuation from a boundary in the presence of caustics. Here, we illustrate reverse-time continuation of boundary data generated by a horizontal plane wave traveling upward through a low velocity lens. The model is plotted in Fig. 1 (left) and consists of a Gaussian low wave speed lens with a variation of $40 \%$ of the peak wave speed of the background model. The initial wave field at $t=0$ is plotted in Fig. 1 (center) and the generated boundary data at $y_{2}=0$ are plotted in Fig. 1 (right).

In Fig. 2 (top), we plot the data obtained after de-recomposition of the time domain finite difference data in Fig. 1 (right) using the wave packet transform. Note that in this de-recomposition step, we can perform denoising, data regularization, or phase-space filtering (dip angle, wave number, location) in the wave packet domain and initiate "beams" [5. We set $t=0$ and monitoring of the points of rank-deficiency of the matrix $W_{1}^{t}$ reveals that we need $N_{s}=4$ time intervals and hence a total of three semigroup decompositions to avoid the formation of caustics in each step of the partial reverse-time continuation. The partitioning of the data in four time slices is indicated with red dashed lines in Fig. 2 (top). 


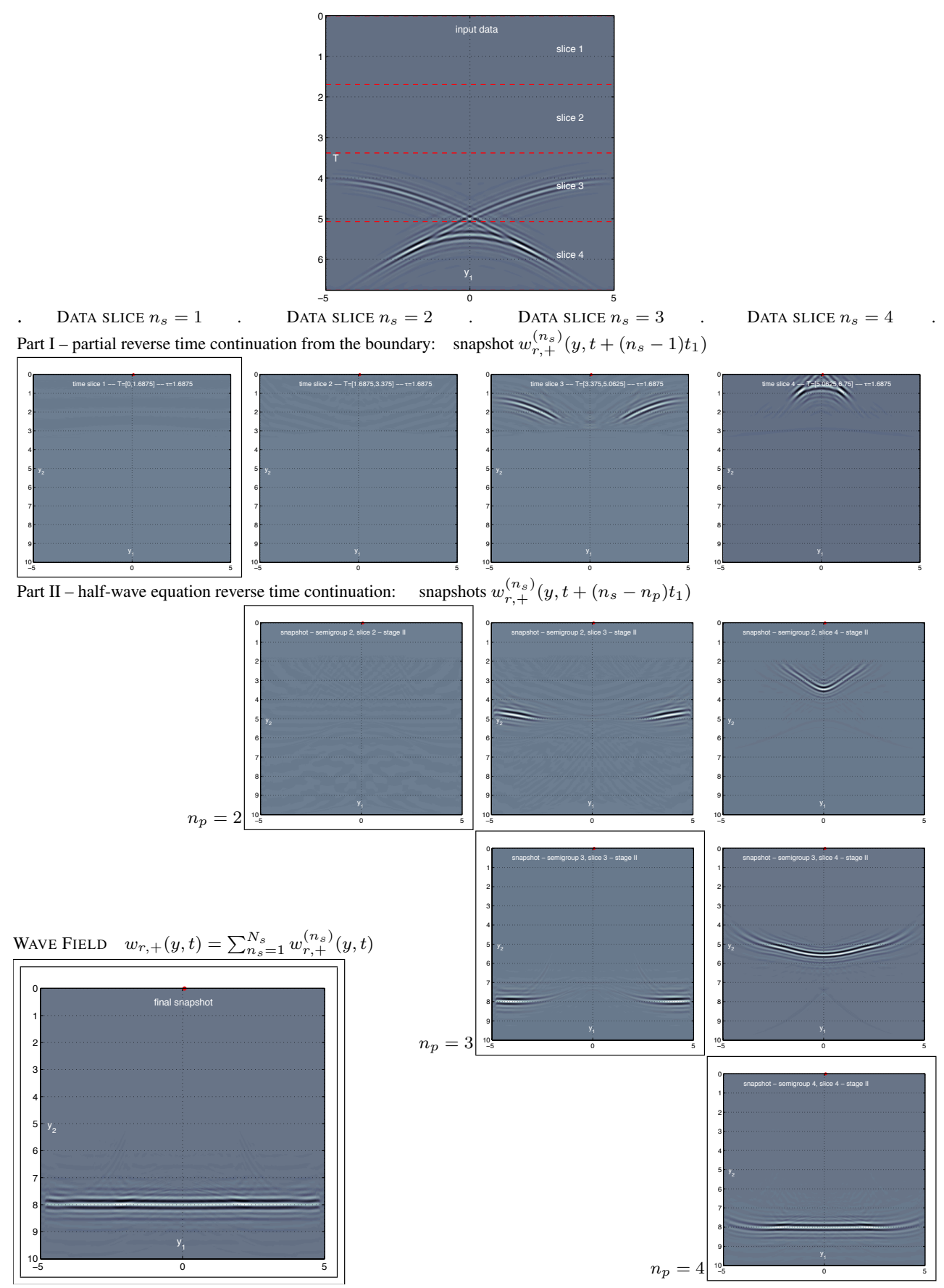

FIG. 2. Top: Partitioning of data in Fig. 1 (right) into 4 time intervals. Center: Reverse-time continuation from the boundary, yielding $w_{r,+}^{\left(n_{s}\right)}\left(y, t+\left(n_{s}-1\right) t_{1}\right), n_{s}=1, \ldots, 4$ (top row); reversetime continued wave fields $w_{r,+}^{\left(n_{s}\right)}\left(y, t+\left(n_{s}-n_{p}\right) t_{1}\right), n_{s}=n_{p}, \ldots, 4$ for $n_{p}=2,3$ and 4 , respectively (rows 2 to 4). Full reverse-time continued wave field $w_{r,+}(y, t=0)$ (bottom left corner). 

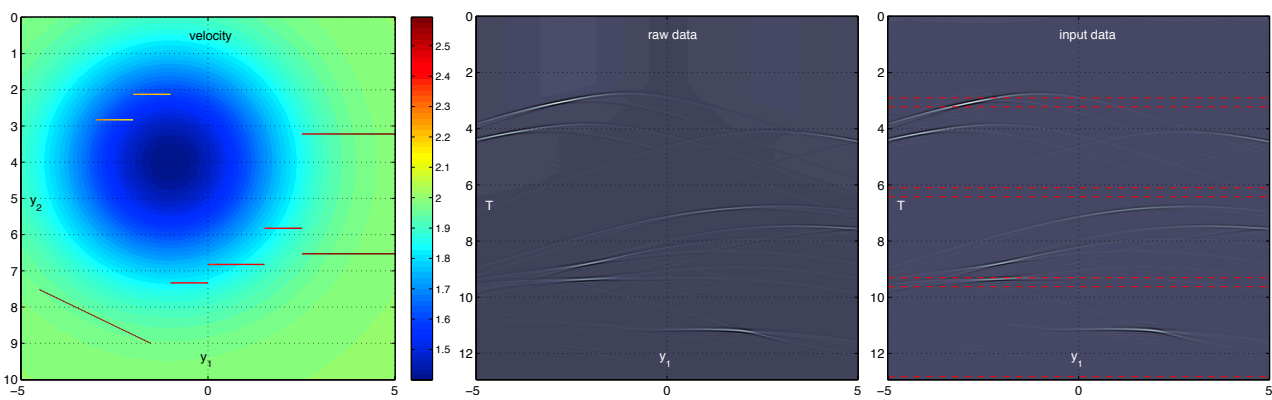

FIG. 3. Imaging of conormal singularities: velocity model with line reflectors (left); time domain finite difference snapshot for source position $\tilde{x}=0$ (center); data after wave packet derecomposition (right), the overlap of the time intervals partitioning the data are indicated by red dashed lines.

The center plots in Fig. 2 show the partial outputs of the reverse-time continuation procedure described in Section 3.4 and illustrate its logic and structure. Each column corresponds with one time interval of the data (from left to right, data slice $n_{s}=1, \ldots, 4$, respectively), and transition from row $i$ to row $i+1$ corresponds with a semigroup re-decomposition and subsequent half wave equation reverse-time continuation step: The top row plots the wave fields $w_{r,+}^{\left(n_{s}\right)}\left(y, t+\left(n_{s}-1\right) t_{1}\right), n_{s}=1, \ldots, 4$, and the second, third, and last rows show $w_{r,+}^{\left(n_{s}\right)}\left(y, t+\left(n_{s}-n_{p}\right) t_{1}\right), n_{s}=n_{p}, \ldots, 4$ for $n_{p}=2,3$ and 4 , respectively (the reverse-time continued wave fields $w_{r,+}^{\left(n_{s}\right)}(y, t=0)$ obtained for the four time intervals are indicated by black frames). The final reversetime continued wave field $w_{r,+}(y, t=0)=\sum_{n_{s}=1}^{N_{s}} w_{r,+}^{\left(n_{s}\right)}(y, t=0)$ is plotted in the bottom left corner of Fig. 2 (black solid double-frame) and reproduces well the initial wave field at time $t=0$ (cf. Fig. 1 (center)). Despite several discrete wave packet transform re-decomposition steps involved in computing the reverse-time continuation (semigroup), the amplitude is accurate. In particular, we note that the edges of the cusp in the data are well focussed.

Note that time intervals 1 and 2 do not contain any significant energy. With the proposed procedure, it is possible to compute only the wave field for time intervals 3 and 4 (requiring no computation time and memory for time slices 1 and 2). Time intervals 1 and 2 have nonetheless been included in Fig. 2 for completeness of the presentation.

5.2. Imaging of conormal singularities. We proceed with a numerical illustration of imaging of conormal singularities by reverse-time continuation from the boundary using the wave packet based computational procedure developed in Section 4. The velocity model is plotted in Fig. 3 (top left). It consists of a decentered Gaussian low velocity (30\% peak contrast with respect to the background velocity) and contains several horizontal line reflectors and one deep tilted line reflector. The (normal incident) reflectivity of the line reflectors varies with location and is documented in Fig. 5 (left). The data are generated using time domain finite difference and a Ricker wavelet with a peak frequency of $7 \mathrm{~Hz}$. The single source is located at the center of the boundary, $\tilde{x}=0$. In Fig. 4. we plot the wave field generated in the subsurface for several time instances (for better visibility, we substracted the wave field that is obtained when the line reflectors are not present). Despite the simplicity of the model, we observe a relatively complicated wave field and, for late 

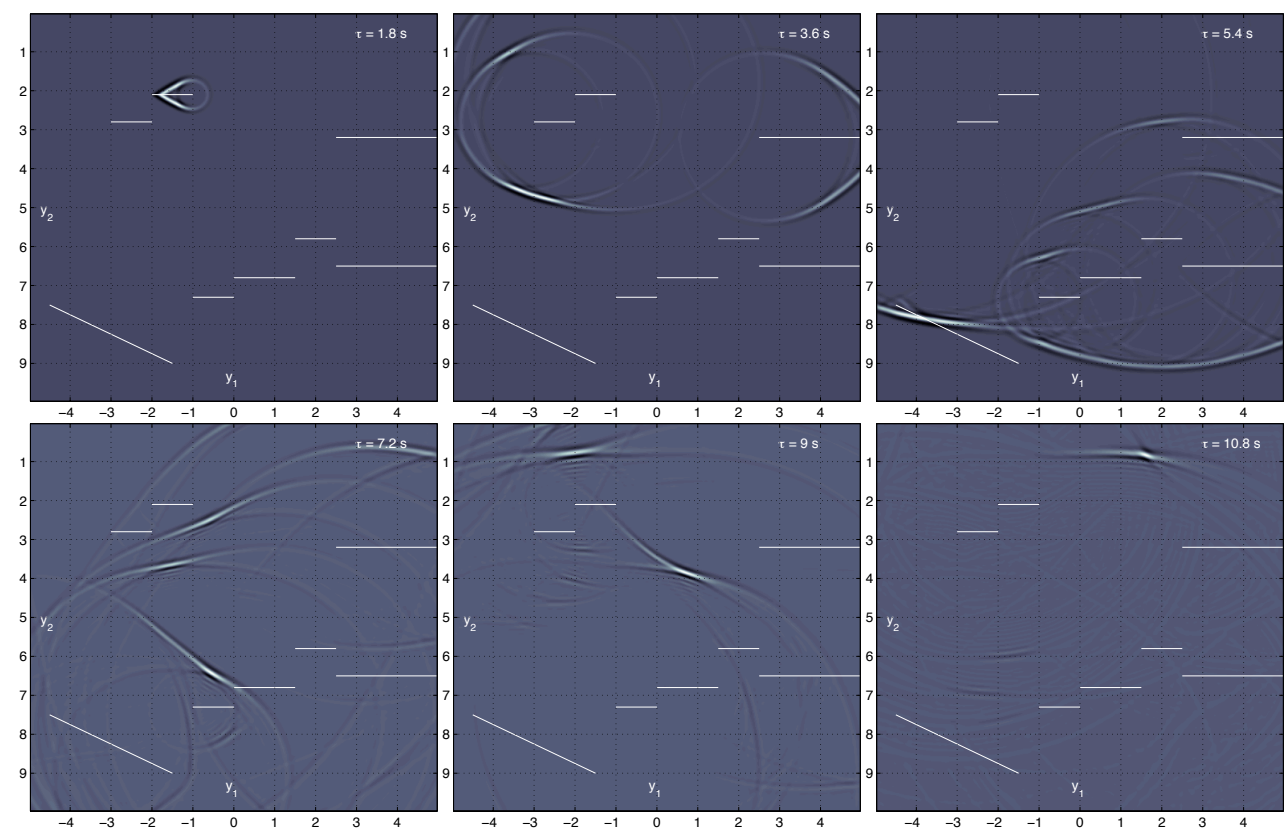

FIG. 4. The wave field generated by a source at $\tilde{x}=0$ for different time instances after subtraction of the reference wave field which is obtained when the line reflectors (indicated in white) are not present.

time instances, the formation of caustics. Also note that artifacts from nonperfectly absorbing boundaries and from multiple reflections, and in particular some numerical dispersion at large times are present in the simulated wave field and consequently also in the data, which we plot in Fig. 3 (center). The data $d_{\Sigma}\left(x^{\prime}, t\right)$ after de-recomposition using the discrete wave packet transform are plotted in Fig. 3 (right). During this derecomposition step, we can also regularize and preprocess the data (denoising, phase space filtering).

In this example, we need $N_{s}=4$ time intervals to avoid conjugate points within each propagation step in the computational procedure described in Section 4 and outlined in Algo. 2 This partitioning into time intervals is detected numerically from the points of rank-deficiency of the matrix $W_{1}^{t}$ of the Hamiltonian system as detailed in [11] and indicated in Fig. 3 (right).

We approximate the source signature with a single delta function at its temporal maximum and compute the source wave field by evaluating the Hamiltonian and Hamilton-Jacobi equations (dynamic ray tracing). In Fig. 6, the partial images and reverse-time continued wave fields produced by the procedure described in Section 4 are plotted, organized according to its hierarchical semigroup structure (cf. Fig. 2). Each column corresponds with one time interval of the data (from left to right, data slice $n_{s}=1, \ldots, 4$, respectively), and transition from (group of) $\operatorname{row}(\mathrm{s}) i$ to (group of) row(s) $i+1$ corresponds with a semigroup re-decomposition and subsequent half wave equation reverse-time continuation and partial imaging step. The top row shows the snapshots $w_{r,+}^{\left(n_{s}\right)}\left(y, t+\left(n_{s}-1\right) t_{1}\right), n_{s}=1, \ldots, 4$ produced by partial reverse-time continuation from the boundary of the 4 data slices (Algo. 2, Part I). The corresponding partial image $\sum_{n_{s}} \Delta^{*\left(n_{s}, t+\left(n_{s}-1\right) t_{1}\right)}(y)$ obtained during this step is plotted in the bottom left corner of Fig. 6. At this stage, data slice 1 is fully reverse-time 


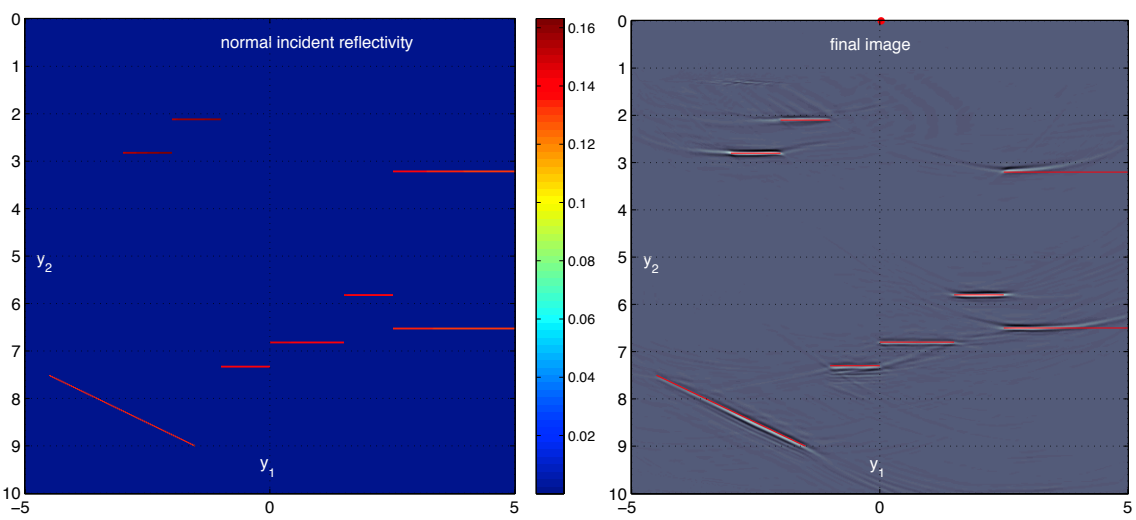

FIG. 5. Normal incident reflectivity of the model (left) and image $\Delta_{d_{\Sigma}}(y)$ (right).

continued $(t=0)$ while data slices 2 to 4 will be further reverse-time continued after a semigroup re-decomposition (and enter Part II of Algo. 2). The second and third groups of rows plot the output of Part II (cf. Algo. 22) of the procedure for $n_{p}=2$ and $n_{p}=3$, respectively: $w_{r,+}^{\left(n_{s}\right)}\left(y, t+\left(n_{s}-n_{p}\right) t_{1}\right)$ (top rows) and $\tilde{\Delta}^{*\left(n_{s}, n_{p}\right)}(y)$ (bottom rows). We stop the semigroup iteration at $n_{p}=3$ because the energy of the data wave fields $w_{r,+}^{\left(n_{s}\right)}\left(y, t+\left(n_{s}-3\right) t_{1}\right)$ has already passed the image region of interest, and further reverse-time continuation would not add any energy to the final image. The partial image contributions of data slices 2 to 4 are plotted in the bottom row of Fig. 6 (second to fourth columns).

Let us finally turn our attention to the image $\Delta_{d_{\Sigma}}(y)=\sum_{n_{s}=1}^{4} \Delta^{*\left(n_{s}, t+\left(n_{s}-1\right) t_{1}\right)}(y)+$ $\sum_{n_{p}=2}^{3} \sum_{n_{s}=n_{p}}^{4} \tilde{\Delta}^{*\left(n_{s}, n_{p}\right)}(y)$, which is plotted in Fig. 5 (right). We observe that all the reflectors are imaged correctly and well focused, regardless of their depth, dip angle and background velocity. Note that we could further focus the image by using the full source signature instead of a delta source approximation. Certain reflectors are partially outside of the zone of illumination (e.g. the two rightmost reflectors at depths $y_{2}=3.2$ and $y_{2}=6.5$ ) and hence produce smiling "tails" caused by the truncation of the wave field in the data (cp. Kirchhoff migration). Similarly, the corners of the line reflectors act as point diffractors and produce tails according to partial illumination and restricted geometry. Note that the ringing artifacts in the data components stemming from the two deepest reflectors are also present in the image - the algorithm produces an image from the data, with its imperfections. This is also the case for the artifact at depth $y_{2}=1.3$ in the image, which results from an imperfectly removed direct arrival (cf. Fig. 3 (right), $\left(y_{1}, t\right)=(3,2.8)$ ).

5.3. Restricted angle transform. Since the proposed boundary source reversetime continuation and imaging procedures rely on the dyadic parabolic decomposition, angular information can be extracted for the source and scattered wave fields. Indeed, for a given frequency box $\hat{\chi}_{\nu, k}$, the incidence angles of the wave fronts are known in each image point. This information can be directly incorporated into the imaging process. Indeed, we can directly generate so-called image gathers in incidence angles (which can be converted to scattering angles), that is, generate images for particular incidence angles. This is illustrated in Figs. 7 and 8 .

In Fig. 7 (second row), we display the images obtained for a single source with the correct velocity model (left column), as in the previous section, and with two wrong velocity models (center and right column, respectively; the corresponding velocity 
models are plotted in the first row of Fig. 7). 


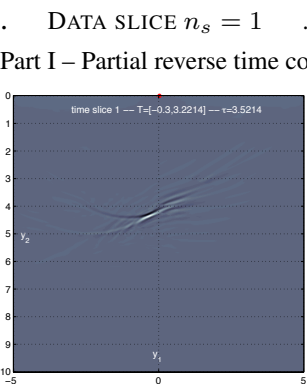

Part II - half-wave equation reverse time continuation:
DATA SLICE $n_{s}=2$

DATA SLICE $n_{s}=3$

snapshots $w_{r,+}^{\left(n_{s}\right)}\left(y, t+\left(n_{s}-1\right) t_{1}\right)$

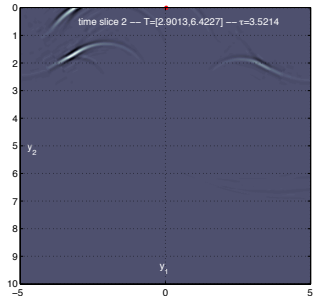

snapshots

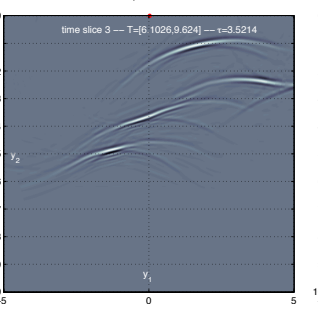

$w_{r,+}^{\left(n_{s}\right)}\left(y, t+\left(n_{s}-n_{p}\right) t_{1}\right)$

partial images $\tilde{\Delta}^{*\left(n_{s}, n_{p}\right)}(y)$
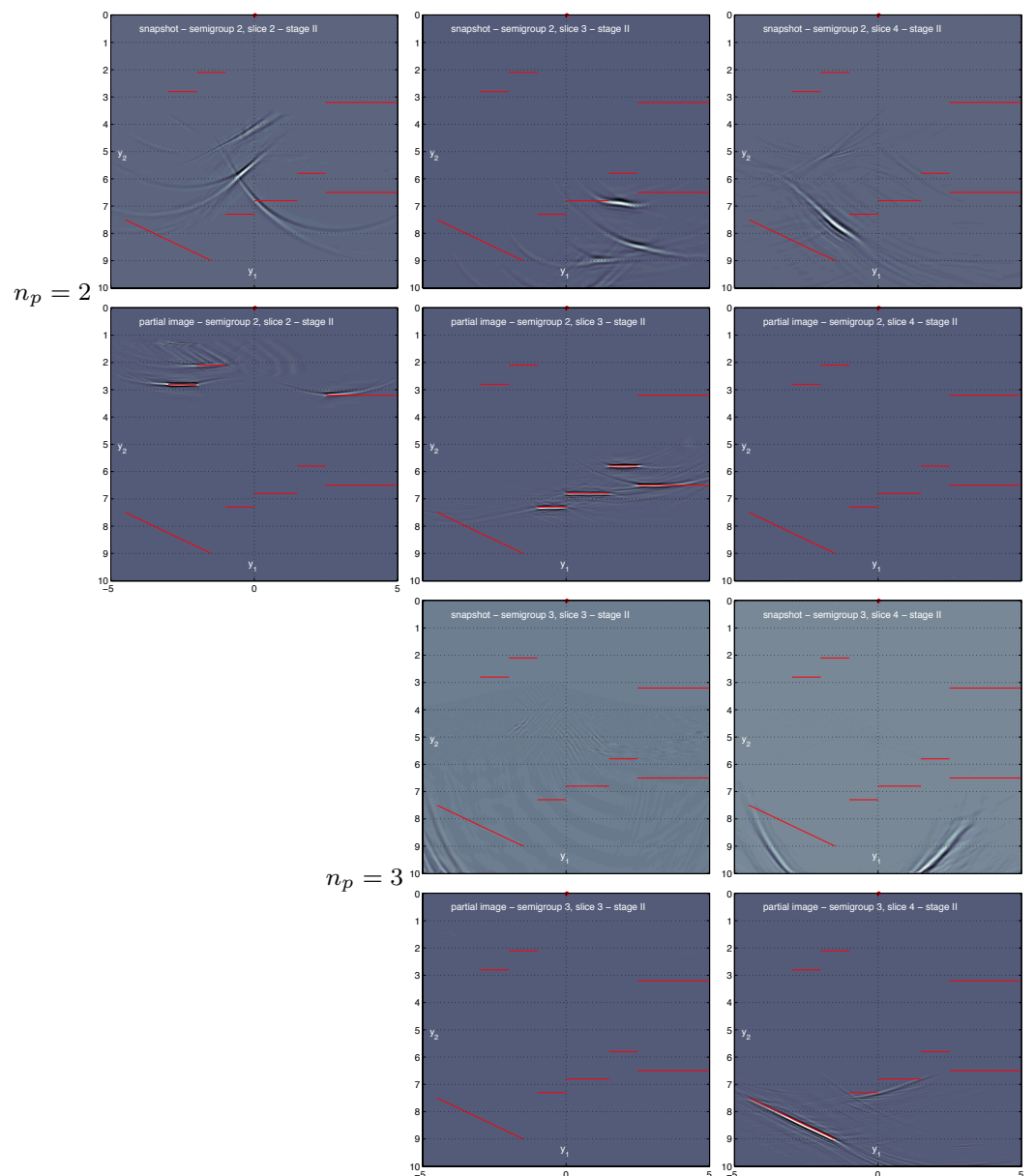

partial image $n_{s}=3$

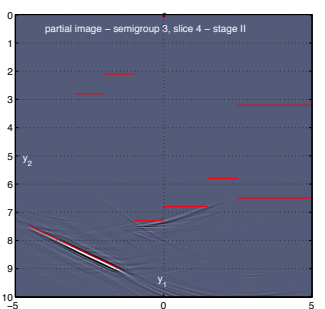

partial image $n_{s}=4$
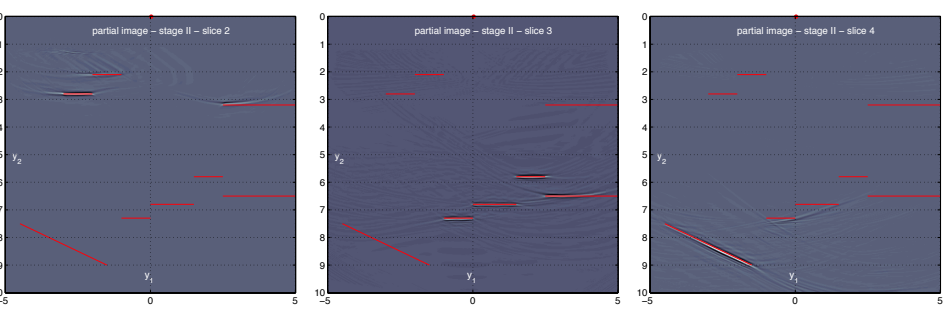

FIG. 6. Partial reverse-time continuation from the boundary of the four time intervals in Fig. 3 (right); reverse-time continuation and imaging following a semigroup re-decomposition of $w_{r,+}^{\left(n_{s}\right)}\left(y, t+\left(n_{s}-n_{p}+1\right) t_{1}\right)$ for $n_{p}=2, n_{s}=2, \ldots, 4$ (center top rows) and for $n_{p}=2, n_{s}=2, \ldots, 4$ (center top rows): snapshots $w_{r,+}^{\left(n_{s}\right)}\left(y, t+\left(n_{s}-n_{p}\right) t_{1}\right)$ and partial images $\tilde{\Delta}^{*}\left(n_{s}, n_{p}\right)(y)$; partial images produced by Part I (bottom row, left) and by Part II for $n_{s}=2,3,4$ (bottom right). 

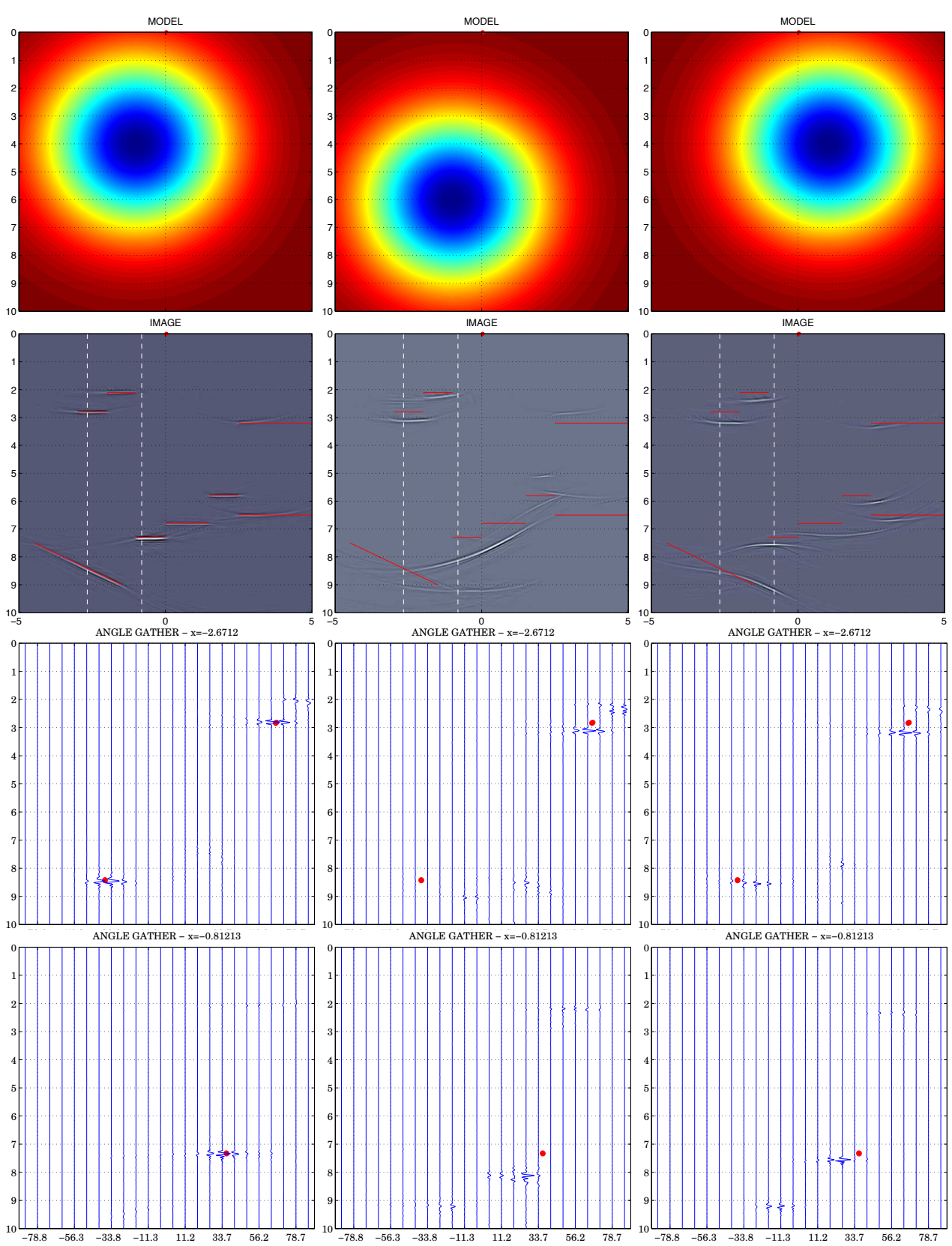

FiG. 7. Velocity models (top row), resulting images (second row) and angle gathers at horizontal positions $x=-2.67$ (third row) and $x=-0.81$ (bottom row): correct velocity model (left column) and wrong velocity models (center and right column). The red dots indicate the specular reflection points for the true velocity model.

In the third and fourth rows of Fig. 7, the images are analyzed as a function of incidence angle at the image points for two distinct boundary locations, respectively. Geometrically, the image of a singularity at one surface location is significant at one incidence angle only; we indicate the incidence angle at specular reflection with a red dot. The finite-frequency content of the wave packets results in a slight spread around these specular reflection angles. For the correct background model, the images are 


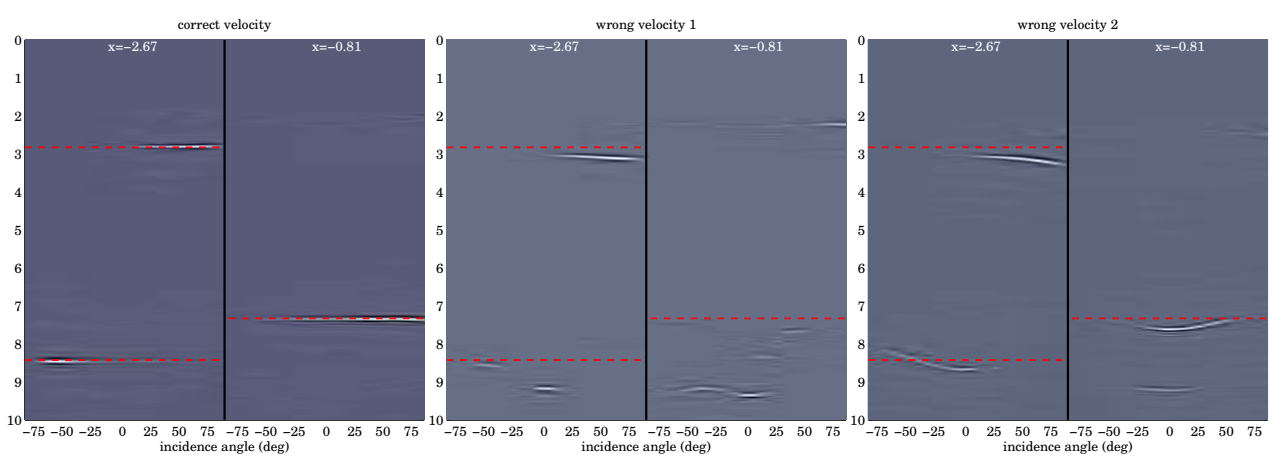

FIG. 8. Image gathers for horizontal positions $x=-2.67$ and $x=-0.81$ as considered in Fig. 7 (left and right half of images, respectively): correct velocity model (left) and wrong velocity models (center and right, respectively). The red dashed lines indicate the depth of the line reflectors.

in phase at the depth of the reflector in the neighborhood of the specular reflection angles, while they are not if the wrong velocity model is used.

We evaluate images of the singularities for multiple sources and rearrange them in terms of local incidence angle (image gather) [13, 34, 35, 37]. In Fig. 8 , the images obtained using the correct (left column) and two wrong (second and third column) velocity models are plotted (the corresponding velocity models are plotted in the first row of Fig. 7). In case the correct background velocity function is used, up to illumination effects, the images generated at different angles are the same; this reflects a redundancy in such data. If we perturb the background by moving the smooth lens, we still obtain coherent images; however, the singularities move with changing incidence angle. This behavior can be exploited to develop a procedure for reflection tomography [26, 7].

6. Discussion. We obtained a representation of RTM in terms of a FIO associated with a canonical graph. We then developed a fast algorithm for solving the wave equation with a boundary source and homogeneous initial conditions using the dyadic parabolic decomposition of phase space, adapting our algorithm for the computation of the action of FIOs associated with canonical graphs [2, which is the key component of its application. We admit the formation of caustics.

Our algorithm is organized by frequency boxes $\hat{\chi}_{\nu, k}$ following the dyadic parabolic decomposition of phase space and yields accuracy $\mathcal{O}\left(2^{-k / 2}\right)$ at frequency scale $k$. We obtain an effective one-step multiscale procedure for reverse-time continuation from the boundary for a given time interval, from $T_{1}$ to $t$, say. In this process, we can apply the imaging condition and obtain a reverse-time-migration imaging algorithm.

While numerical illustrations have been devised here for dimension 2, the concepts and computational procedures are valid for arbitrary dimension.

In the presence of conjugate points, we split the time interval for reverse-time continuation into a sequence of smaller time intervals and reverse-time continue partial wave fields subsequently for these time intervals using the semigroup property of the RTM operator. Numerically, this implies one discrete wave packet transform redecomposition of the wave fields for each transition point from one time interval to another. After the first semigroup re-decomposition, reverse-time continuation essentially reduces to the evaluation of the wave equation for the propagation of an initial wave field, and any of the algorithms developed in 2 could be used as a computational basis. Here, we proposed a "box algorithm" due to its favorable computational complexity and practical accuracy. 
The computational complexity of our algorithm is of the order $\mathcal{O}\left(N^{n} \log (N)\right)$ per frequency box for each semigroup step for an $n$-dimensional grid of side length $N$. It arises essentially from the complexity of the unequally spaced FFTs involved in the box algorithm (cf. 2] for details). Computations for each individual box are independent and hence embarrassingly parallel. The computational cost of RTM imaging is roughly twice that of reverse-time continuation of the wave field from the boundary because of the additional unequally spaced FFTs that need to be evaluated for producing the image. Note that with the exception of the source wave field travel times and amplitudes and one single snapshot during each semigroup re-decomposition, our procedure does not require the computation and storage of snapshots. Its computational and memory requirements are therefore of the order of the one-step evaluation of Cauchy initial value problems for evolution equations in 2 .

Evaluation of the RTM operator for all wave packets associated with a given frequency boxes $\hat{\chi}_{\nu, k}$ at once requires the existence of a homogeneous boundary layer near the acquisition surface. When the wave speed is not constant near the boundary, we need to localize computations and either introduce a partitioning of the acquisition surface or use wave packets as individual local data quanta, the latter yielding a wave packet based procedure at the price of increased computational complexity with respect to a frequency box driven algorithm.

The total number of frequency boxes is $\mathcal{O}\left(N^{(n-1) / 2}\right)$, this number can be slightly reduced by replacing frequency boxes (tiles) with wedges as in [9, yet at the price of losing the connection to the data wave packets. Depending on the data and the imaging target, not all boxes need to be computed. Indeed, our algorithm provides phase-space localized control for the data (scale, orientation, position of the data wave packets) as well as the image (scale, orientation and position of reverse-time continued data wave packets; full angular information such as scattering angle, and reflector dip angle). Together with the fact that only a few time steps need to be computed for producing an image, this makes our algorithm particularly attractive for (limited aperture) array data, partial imaging and target-oriented imaging. An additional asset of our approach is that incident angles of wave fronts can also be imaged, enabling the straightforward application of restricted angle transforms.

We note that by viewing wave packets as localized plane waves, our method can be related to plane-wave and beam-wave migration [5]. Here, we can construct "beams" as reverse-time continued data wave packets based on phase-space localized paraxial approximation in geodesic coordinates. In the context of imaging with ambient noise using body waves and beamforming [21], one generates a cross correlation matrix between two distant receiver arrays at which ambient noise generated data are obtained, and one takes inner products with wave packets and can enhance particular wave constituents prior to applying the imaging operator.

Reverse-time continuation from the boundary can in principle be generalized to extended imaging using multisource data based on downward continuation [27. The corresponding evolution equation replacing (2.6) can be found in [15, Eq. (17)]. In this case, the evolution equation is defined in $(2 n-1)$-dimensional extended space.

\section{REFERENCES}

[1] U. Albertin, D. Yingst, H. Jaramillo, W. Wiggins, C. Chapman, and D. Nichols, Towards a hybrid raytrace-based beam/wavefield-extrapolated beam migration algorithm, SEG Technical Program Expanded Abstracts, (2002), pp. 1344-1347. 
[2] F. Andersson, M. De Hoop, And H. Wendt, Multiscale discrete approximation of Fourier integral operators, Multiscale Model. Simul., 10 (2012), pp. 111-145.

[3] K. Baysal, D. Kosloff, and J. Sherwood, Reverse time migration, Geophysics, 48 (1983), pp. 1514-1524.

[4] B. L. Biondi, 3D seismic imaging, Society of Exploration Geophysicists, 2006.

[5] S. Brandsberg-Dahl and J. Etgen, Beam-wave migration, European Association of Geoscientists and Engineers, Extended Abstracts, 23 (2003), pp. 969-972.

[6] V. BRYtiK, M. V. DE HoOp, AND R. D. VAN DER HILst, Elastic-wave inverse scattering based on reverse time migration with active and passive source reflection data, Inverse Problems and Applications: Inside Out II, 60 (2013), p. 411.

[7] S. Burdick, M. De Hoop, R. VAn Der Hilst, And S. Wang, Rtm-based reflection tomography using teleseismic free surface reflected phases, Geophys. J. Int., (2013, submitted).

[8] E. Candès, L. Demanet, D. Donoho, And L. Ying, Fast discrete curvelet transforms, SiAM Multiscale Model. Simul., 5 (2006), pp. 861-899.

[9] E. Candès, L. Demanet, and L. Ying, A fast butterfly algorithm for the computation of fourier integral operators, Multiscale Modeling \& Simulation, 7 (2009), pp. 1727-1750.

[10] J. F. Claerbout, Imaging the Earth's Interior, Blackwell Scientific Publications, Inc., 1985.

[11] M. V. De Hoop, G. Uhlmann, A. Vasy, And H. Wendt, Multiscale discrete approximations of fourier integral operators associated with canonical transformations and caustics, Multiscale Modeling \& Simulation, 11 (2013), pp. 566-585.

[12] L. Demanet and L. Ying, Fast wave computation via fourier integral operators, Mathematics of Computation, 81 (2012), pp. 1455-1486.

[13] T. Dickens And G. WinBow, Rtm angle gathers using poynting vectors, in Expanded Abstracts, Society of Exploration Geophysicists, 2011, pp. 3109-3113.

[14] A. Duchkov, F. Andersson, And M. DE Hoop, Discrete almost symmetric wave packets and multi-scale representation of (seismic) waves, IEEE T. Geosci. Remote Sensing, 48 (2010), pp. 3408-3423.

[15] — Extended isochron rays in prestack depth (map) migration, Geophysics, 75 (2010), pp. S139-S150.

[16] A. Duchkov, M. De Hoop, And A. SÁ Barreto, Evolution-equation approach to seismic image, and data, continuation, Wave Motion 45 (2008), pp. 952-969.

[17] J. DuistermaAt, Fourier integral operators, Birkhäuser, Boston, 1996.

[18] J. Li AND D. YuEn, Mid-mantle heterogeneities associated with izanagi plate: Implications for regional mantle viscosity, Earth and Planetary Science Letters, 385 (2013), pp. 37-144.

[19] G. McMechan, Migration by extrapolation of time-dependent boundary values, Geophys. Prosp., 31 (1983), pp. 413-420.

[20] T. Op'T Root, C. StOLK, AND M. De Hoop, Linearized inverse scattering based on seismic reverse-time-migration, J. Math. Pures Appl., in print (2012).

[21] P. Poli, M. Campillo, AND H. Pedersen, Body-wave imaging of earth?s mantle discontinuities from ambient seismic noise, Science, 338 (2012), pp. 1063-1065.

[22] P. SAva And S. Fomel, Riemannian wavefield extrapolation, Geophysics, 70 (2005), pp. T45T56.

[23] P. S. Schultz And J. W. C. Sherwood, Depth migration before stack, Geophysics, 45 (1980), pp. 376-393.

[24] G. Shan ANd B. Biondi, Imaging overturned waves by plane-wave migration in tilted coordinates, SEG Technical Program Expanded Abstracts, 23 (2004), pp. 969-972.

[25] H. Smith, A parametrix construction for wave equations with $c^{1,1}$ coefficients, Ann. Inst. Fourier, Grenoble, 48 (1998), pp. 797-835.

[26] C. Stolk And M. De Hoop, Seismic inverse scattering in the downward continuation approach, Wave Motion, 43 (2006), pp. 579-598.

[27] C. C. Stolk And M. V. DE Hoop, Modeling of seismic data in the downward continuation approach, SIAM J. Appl. Math., 65 (2005), pp. 1388-1406.

[28] R. Sun and G. McMechan, Scalar reverse-time depth migration of prestack elastic seismic data, Geophysics, 66 (2001), pp. 1519-1527.

[29] W. W. Symes, Topical review: The seismic reflection inverse problem, Inverse Problems, 25 (2009), p. 123008.

[30] M. E. Taylor, Pseudodifferential Operators, Princeton University Press, Princeton, 1981.

[31] L. Vinnik, M. Kato, and H. Kawakatsu, Search for seismic discontinuities in the lower mantle, Geophys. J. Int., 147 (2001), pp. 41-56.

[32] D. Whitmore, Iterative depth migration by backward time propagation, in Expanded Abstracts, Society of Exploration Geophysicists, 1983, pp. 382-385.

[33] N. Whitmore, An imaging hierarchy for common angle plane wave seismograms, $\mathrm{PhD}$ thesis, 
University of Tulsa, 1995.

[34] K. Yoon, M. GUO, J. CAI, AND B. WANG, 3D RTM angle gathers using wave propagation direction and dip, in Expanded Abstracts, Society of Exploration Geophysicists, 2011, pp. 3136-3139.

[35] K. Yoon, K. Marfurt, and W. Starr, Challenges in reverse-time migration, in Expanded Abstracts, Society of Exploration Geophysicists, 2004, pp. 1057-1060.

[36] - Reverse-time migration using the poynting vector, Exploration Geophysics, 37 (2006), pp. 102-107.

[37] Q. Zhang And G. McMechan, Angle-domain common-image gathers extracted from reversetime migrated images in isotropic and elastic media, in Expanded Abstracts, Society of Exploration Geophysicists, 2011, pp. 3130-3135. 